\title{
Discrete-Time Mixing Receiver Architecture for RF-Sampling Software-Defined Radio
}

\author{
Zhiyu Ru, Member, IEEE, Eric A. M. Klumperink, Senior Member, IEEE, and Bram Nauta, Fellow, IEEE
}

\begin{abstract}
A discrete-time (DT) mixing architecture for RF-sampling receivers is presented. This architecture makes RF sampling more suitable for software-defined radio (SDR) as it achieves wideband quadrature demodulation and wideband harmonic rejection. The paper consists of two parts. In the first part, different downconversion techniques are classified and compared, leading to the definition of a DT mixing concept. The suitability of CT-mixing and RF-sampling receivers to SDR is also discussed. In the second part, we elaborate the DT-mixing architecture, which can be realized by de-multiplexing. Simulation shows a wideband $90^{\circ}$ phase shift between I and Q outputs without systematic channel bandwidth limitation. Oversampling and harmonic rejection relaxes $R F$ pre-filtering and reduces noise and interference folding. A proof-of-concept DT-mixing downconverter has been built in $65 \mathrm{~nm}$ CMOS, for 0.2 to $0.9 \mathrm{GHz}$ RF band employing 8-times oversampling. It can reject 2nd to 6th harmonics by $40 \mathrm{~dB}$ typically and without systematic channel bandwidth limitation. Without an LNA, it achieves a gain of -0.5 to $2.5 \mathrm{~dB}$, a DSB noise figure of 18 to $20 \mathrm{~dB}$, an IIP3 $=+10 \mathrm{dBm}$, and an IIP2 $=+53 \mathrm{dBm}$, while consuming less than $19 \mathrm{~mW}$ including multiphase clock generation.
\end{abstract}

Index Terms-Sampling, RF sampling, sampling receiver, oversampling, mixing, discrete-time mixing, continuous-time mixing, software-defined radio, SDR, software radio, SWR, harmonic rejection, anti-aliasing, phase shift, quadrature, demodulation, downconversion, downconverter, de-multiplexing, de-multiplexer, wideband, wideband sampling, receiver, wideband receiver, interference, CMOS, system-on-chip, SoC.

\section{INTRODUCTION}

$\mathbf{R}$ F-SAMPLING receivers have recently drawn both academic [1], [2] and industrial interests [3]-[6]. They sample the signal early in the receiver chain before or simultaneously with downconversion, instead of after downconversion as done in conventional continuous-time (CT) mixing receivers. Direct RF-sampling provides the potential to move A/D converters (ADC) closer to antenna aiming at software radio (SWR) or software defined radio (SDR). As initially proposed by Mitola [7] and BellSouth [8], the ADC in an ideal SWR operates on the RF signal, while in a (more practical) SDR the ADC may be located at IF, after frequency downconversion, still achieving certain flexibility. Reconfigurability by software should allow it to support different wireless standards in one device, bringing cost and size reductions. SDR might also allow

Manuscript received August 21, 2009; revised April 29, 2010; accepted May 05, 2010. Date of current version August 25, 2010. This paper was approved by Associate Editor Bevan Baas.

The authors are with the IC Design Group, Department of Electrical Engineering, University of Twente, 7500 AE Enschede, The Netherlands (e-mail: z.ru@ewi.utwente.nl).

Color versions of one or more of the figures in this paper are available online at http://ieeexplore.ieee.org.

Digital Object Identifier 10.1109/JSSC.2010.2053860 for upgradable radios and cognitive radio (CR), to improve the efficiency of utilizing scarce spectrum resources.

Several CMOS ICs have recently been published with features suitable for SDR receivers. Reference [9] focuses on flexible baseband filters using switched-capacitor circuits and [10] on the programmability of parameters such as gain, noise figure (NF), linearity, and power consumption. Reference [11] proposes techniques to mitigate out-of-band interference with less dedicated RF pre-filtering. All these receivers [9]-[11] use wideband front-ends based on CT mixing. On the other hand, traditional RF sampling [1]-[6] is not directly suitable for SDR [12], as it provides quadrature demodulation and harmonic rejection over a limited channel bandwidth (BW), limiting SDR flexibility.

This paper proposes a discrete-time (DT) mixing architecture [13], featuring wideband quadrature mixing for I/Q demodulation and image rejection, and wideband ${ }^{1}$ harmonic rejection to suppress wideband interference. To verify the concept, we present a downconverter in $65 \mathrm{~nm}$ CMOS targeting CR applications in the television broadcasting band [14]. Wideband features can be useful there to exploit free spectrum segments distributed over a wide band in CR applications.

Compared to [13], this paper proposes a way to classify different downconversion techniques to clarify distinctions, and discusses different receiver architectures for SDR. Moreover, it describes the DT mixing concept and circuit implementation in detail, and presents new measurements obtained from packaged chips ([13] used wafer probing). This work focuses on the DT-mixing downconverter, while [15] focuses on the front-end tunable $L C$ filter and the LNA.

The classification and comparison of frequency downconversion techniques is described in Section II, showing how DT mixing is different. Section III further explains the DT mixing concept and how it can be used to reduce aliasing. To verify the concept, a specific implementation in $65 \mathrm{~nm}$ CMOS is presented in Section IV, followed by Section V discussing the measurement results. Conclusions are drawn in Section VI.

\section{Classification AND COMPARISON OF FREQUENCY DOWNCONVERSION TECHNIQUES}

Different frequency downconversion techniques have been proposed for radio receivers, most notably mixing and sampling. We would like to compare them and answer questions such as: what are the fundamental distinctions among them? Does early sampling in a receiver chain bring additional flexibility? Does sampling suffer more from clock jitter compared to mixing?

\footnotetext{
${ }^{1}$ Twice "wideband" refers to wide channel bandwidth, while "wideband interference" refers to both the RF and channel bandwidth.
} 
TABLE I

ClassifiCATION OF FREQUENCY DOWNCONVERSION TECHNIQUES*

\begin{tabular}{|c|c|c|c|}
\hline Principle Input & Analog-CT & Analog-DT & Digital \\
\hline $\begin{array}{c}\text { Mixing } \\
\text { (observation rate } \\
\text { does not change) }\end{array}$ & CT Mixing ${ }^{1}$ & $\begin{array}{l}\text { DT Mixing² } \\
\text { (proposed) }\end{array}$ & $\begin{array}{l}\text { Digital } \\
\text { Mixing }^{3}\end{array}$ \\
\hline $\begin{array}{l}\text { Sampling } \\
\text { (observation rate } \\
\text { does change) }\end{array}$ & $\begin{array}{l}\text { CT-to-DT } \\
\text { Sampling }{ }^{2}\end{array}$ & $\begin{array}{l}\text { DT Re- } \\
\text { sampling }{ }^{2}\end{array}$ & $\begin{array}{l}\text { Digital Re- } \\
\text { sampling }^{3}\end{array}$ \\
\hline
\end{tabular}

${ }^{1} \mathrm{CT}$-mixing receiver ${ }^{2} \mathrm{RF}$-sampling receiver ${ }^{3}$ Ideal SWR receiver *Based on the type of input signal (continuous/discrete in time/amplitude) and the downconversion principle (mixing/sampling); commonly used receiver names are also indicated by superscripts (see also Fig. 2), showing that RF sampling fits three classes.

To compare downconversion techniques, we propose a classification in Section II-A. This also leads to the definition of the DT-mixing technique, which is the main subject of Sections III to V. Section II-B compares mixing and sampling, stressing their fundamental distinction. Based on the classification, receiver architectures and their suitability for SDR are discussed in Section II-C.

\section{A. Classification of Downconversion Techniques}

Table I classifies frequency downconversion techniques in two respects. The columns are defined by the input signal domain, i.e., continuous-time (CT) versus discrete-time (DT) and continuous-amplitude versus discrete-amplitude. This results in three columns: analog-CT (CT and continuous-amplitude), analog-DT (DT and continuous-amplitude), and digital (DT and discrete-amplitude). ${ }^{2}$ The rows differ in downconversion principle, i.e., mixing or sampling, where downconversion by sampling is due to aliasing. Thus:

1) For an analog-CT input signal, the downconversion can be realized by either CT mixing or CT-to-DT sampling. Please note that a sampler may or may not do downconversion (aliasing), depending on Nyquist criterion.

2) For an analog-DT input signal, DT mixing but also DT re-sampling can realize downconversion. Note that DT re-sampling has many uses, e.g., decimation by down-sampling or interpolation by up-sampling. Here the purpose is to exploit the aliasing effect associated with re-sampling for frequency conversion.

3) For a digital input signal, downconversion can be done by digital mixing or digital re-sampling.

While all the other five techniques in Table I have been discussed in literature, to the authors' knowledge, we were the first to propose and implement the concept of DT mixing in a receiver [13]. References [1]-[6] apply CT-to-DT sampling for downconversion, while [9]-[11] and [18]-[20] apply CT mixing. DT re-sampling (decimation) has also been used in [1] for a second downconversion.

${ }^{2} \mathrm{~A}$ column for CT discrete-amplitude signals could also be added, but we omitted it for simplicity and because we are not aware of any radio example operating in this domain, although some work [16], [17] explores the properties of CT DSP systems.

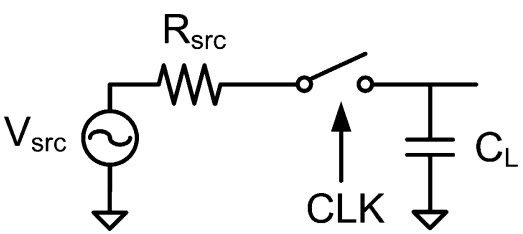

Fig. 1. A general switching system for mixer or sampler.

Sometimes, classification is difficult. For instance, the circuit in Fig. 1 has been presented as a passive mixer $^{3}$ [18]-[20] but also as a sampler [1], [2]. The next section discusses mixing and sampling in a bit more detail to assist classification.

\section{B. Fundamental Distinction Between Mixing and Sampling: Observation Rate Changes or Not}

Both mixing and sampling are often explained as a multiplication in the time domain or convolution in the frequency domain. However, there is a fundamental distinction, as discussed below. When CT-to-DT sampling is written as a multiplication of a CT input signal $x(t)$ with a Dirac comb $\delta_{T}(t)=$ $\Sigma \delta\left(t-n \cdot T_{s}\right)$, where $\mathrm{n}$ is an integer among $[-\infty,+\infty]$, the sampled signal $x_{s}(t)$ and its Fourier transform become

$$
\begin{aligned}
x_{s}(t) & =x(t) \cdot \delta_{T}(t) \stackrel{\text { Fourier }}{\leftrightarrow} \\
X_{s}(f) & =X(f) \otimes \frac{1}{T_{s}} \sum_{n=-\infty}^{+\infty} \delta\left(f-n f_{s}\right) \\
& =\frac{1}{T_{s}} \sum_{n=-\infty}^{+\infty} X\left(f-n f_{s}\right) .
\end{aligned}
$$

where $T_{s}$ and $f_{s}$ are the period and the frequency of the sampling clock. $X(f)$ and $X_{s}(f)$ are Fourier transforms of $x(t)$ and $x_{s}(t)$ respectively. Equation (1) is commonly used to describe ideal sampling, but the scaling factor $1 / T_{s}$ is often neglected. This scaling factor suggests a "gain" of $1 / T_{s}$ with a unit of " $\mathrm{Hz}$ ", which seems strange. This issue can be resolved by realizing that (1) is only half of the story. Sampling is more than just multiplication; namely also CT-to-DT conversion.

The signals $x(t), \delta_{T}(t)$, and $x_{s}(t)$ are all defined in the CT domain. If we convert $x_{s}(t)$ into DT-domain as $x_{s}(k)$ and apply a DT Fourier transform, we get a frequency-domain representation with continuous but normalized frequency axis, i.e., $f / f_{s}$. Defining ratio $r=f / f_{s}$, we can obtain the frequency-domain representation on the r-axis by substituting $f=r \cdot f_{s}$ into (1):

$$
\begin{aligned}
X_{s}\left(r \cdot f_{s}\right) & =X\left(r \cdot f_{s}\right) \otimes \frac{1}{T_{s}} \sum_{n=-\infty}^{+\infty} \delta\left(r \cdot f_{s}-n \cdot f_{s}\right) \\
& =X\left(r \cdot f_{s}\right) \otimes \sum_{n=-\infty}^{+\infty} \delta(r-n) .
\end{aligned}
$$

Note that the factor $1 / T_{s}$ disappears in (2), due to the scaling property of the $\delta$-function. If we define $Y_{s}(r)=X_{s}\left(r \cdot f_{s}\right)$ and $Y(r)=X\left(r \cdot f_{s}\right)$, then (2) can be written as

$$
Y_{s}(r)=Y(r) \otimes \sum_{n=-\infty}^{+\infty} \delta(r-n)=\sum_{n=-\infty}^{+\infty} Y(r-n) .
$$

${ }^{3}$ For a current-driven mixer, a resistor in parallel with $C_{L}$ would be desired to define the voltage conversion gain from RF to baseband. But if the mixer is driven by a voltage source such as in Fig. 1, such a resistor is not necessary. 
$Y_{s}(r)$ is the frequency-domain representation of $x_{s}(k)$ and $Y(r)$ is equivalent to $X(f)$ but on the r-axis. Comparing (3) to (1), we see that in the true DT domain, the scaling farctor $1 / T_{s}$ has disappeared and the gain of an ideal sampler is 1 , as expected. Therefore, the step of CT-to-DT conversion is crucial for a sampling operation.

DT signal has a finite "observation rate" (same as sample rate for the DT case) which is inversely proportional to the sampling interval $\Delta T$. For the sake of the classification, a CT signal can be considered as a limiting case of a DT signal with $\Delta T \rightarrow 0$, i.e., its "observation rate" goes to infinity. Thus, we can view CT-to-DT conversion as a way to reduce the observation rate from infinite (CT signal) to finite (DT signal) and CT-to-DT sampling nicely fits in one row with DT re-sampling and digital re-sampling (second row of Table I). They sample or re-sample the input signal so to change $\Delta T$ between samples and hence the observation rate. In contrast, all the mixing techniques translate the signal frequency but do not change $\Delta T$ nor the observation rate. For a CT mixer, both input and output signals are continuous in time, i.e., both have an infinite observation rate. For a DT mixer or a digital mixer, the input and the output signals also have the same (finite) observation rate.

This time-domain property can serve as an important distinction between mixing and sampling. For example, for Fig. 1 there is a simple distinction depending on how the output signal is used. If the output signal is used continuously over all time, then the circuit is a CT mixer. However, if the output signal is only evaluated at discrete time points, e.g., only at the end of each LO cycle, then the circuit is a CT-to-DT sampler. Thus, the distinction is not in the circuit itself but in the way of interpreting/using the output, i.e., whether the observation rate changes or not from input to output.

Note that we discussed a CT-to-DT sampling above, without "hold effect" 4 which otherwise means a CT output. Therefore, in this paper, we will use the letter " $\mathrm{S}$ " as the symbol for sampler but not "S/H".

\section{Receiver Architectures for SDR}

We will now discuss different ways to implement frequency downconversion in receiver architectures and discuss their suitability to SDR applications. Fig. 2 shows three simplified architectures, each consisting of a mixer, low-pass filter (LPF), sampler (S) and quantizer (Q). Other blocks such as amplifiers and LOs are also important but omitted here for figure clarity. Comparing Fig. 2(a), (b) and (c), the mixer-LPF combination shifts to the right and the sampler/quantizer to the left, representing the trend to move the sampler closer to the receiver input and apply RF-sampling.

Fig. 2(a) shows a traditional CT-mixing receiver with frequency conversion in the $\mathrm{CT}$ domain, which is commonly used today for zero-IF or low-IF receivers. In contrast, in Fig. 2(c) frequency conversion is done in the digital domain, after both

\footnotetext{
${ }^{4}$ On the other hand, a "hold effect" can provide DT-to-CT conversion, e.g., used as the reconstruction filter in a digital-to-analog converter. Effectively a hold increases the observation rate from finite (DT) to infinite (CT). Interestingly, applying a hold following a sampler, which makes a sample-and-hold $(\mathrm{S} / \mathrm{H})$ together, introduces a coefficient proportional to the holding time, which may also cancel the factor $1 / T_{s}$ in (1).
}

(a)

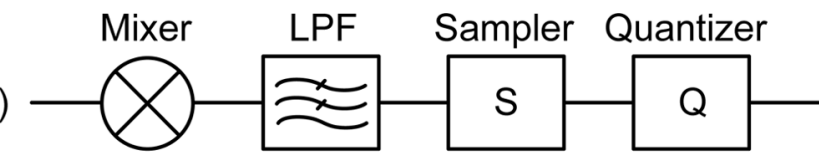

(b)

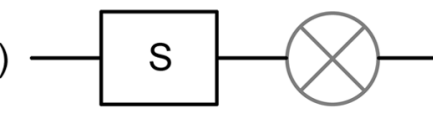

(c)

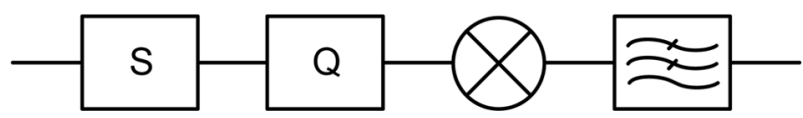

Fig. 2. Three (simplified) receiver architectures: (a) CT-mixing receiver; (b) RF-sampling receiver; (c) ideal software radio (SWR) receiver; showing a trend to move the "mixer-LPF" to the back-end.

sampling and quantization (A/D conversion). Here either a digital mixer or digital re-sampler can be used for frequency downconversion. This type of receiver fits in the last column of Table I and is indicated as "ideal SWR receiver".

Fig. 2(b) shows a sampler followed by a mixer, in front of the quantizer. As the CT-to-DT sampler receives RF signals, this architecture is often called an "RF-sampling receiver" in literature. This name includes three sub-classes as shown in Table I. In traditional RF-sampling receivers [1]-[6], the DT mixer in Fig. 2(b) (shown in grey) is missing since the downconversion was realized using the CT-to-DT sampler itself or a following DT re-sampler. Also the ideal SWR receiver samples signal at RF but we do not call it an "RF-sampling receiver" in accordance with literature.

The ideal SWR receiver [Fig. 2(c)] offers maximum flexibility, as it can select and process any band or channel in the digital domain. On the other hand, the CT-to-DT sampler (S) in an ideal SWR receiver does not translate desired signals in frequency, but only does so in the digital domain. Therefore, signal processing requirements are huge and $\mathrm{ADC}$ has to convert the full RF-spectrum of interests directly into the digital domain which is usually not feasible [21]. The RF-sampling receivers implemented in [1]-[6] still down-convert and select the desired channel in the analog domain. Therefore, the main asset of an ideal SWR receiver, i.e., flexibility, is not inherently shared by RF-sampling receivers. Still, RF-sampling receivers [Fig. 2(b)] as well as CT-mixing receivers [Fig. 2(a)] can be adapted for some degree of flexibility and software control, to make them suitable for SDR applications. However, there is no apparently a priori preference for one of the two.

A common concern for RF-sampling receivers is the clock jitter. It can be shown [22] that the jitter induced error for CT-mixing is proportional to the LO frequency $f_{\mathrm{LO}}$, while for RF-sampling it is proportional to the RF input frequency $f_{\mathrm{RF}}$. For zero-IF or low-IF receivers, $f_{\mathrm{LO}}$ and $f_{\mathrm{RF}}$ are almost equal, and hence the difference in jitter requirement between CT-mixing and RF-sampling receivers is small [22]. The feasibility of RF-sampling receivers for practical use has been verified by [3]-[6].

Compared to CT-mixing receivers, $\mathrm{RF}$-sampling receivers may require extra complexity, due to RF-related baseband sample rate [12] and extensive clock control for the switched-capacitor (SC) circuits used for DT operation. However, RF sampling may also offer advantages on the compatibility to CMOS 


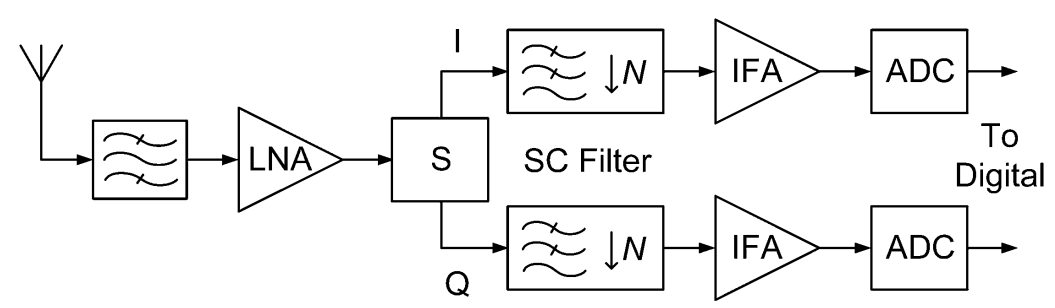

(a)

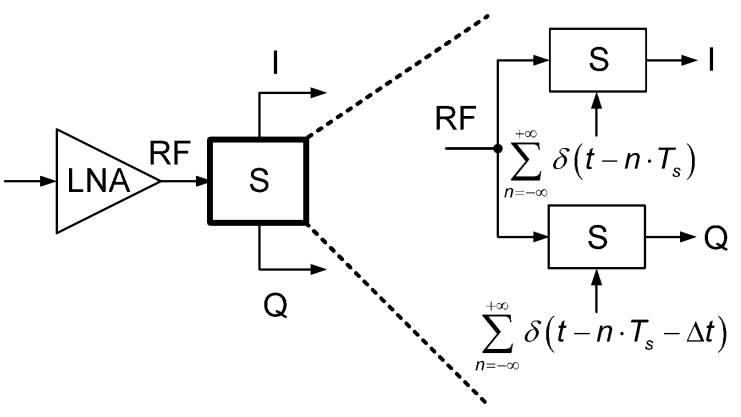

(b)

Fig. 3. (a) Block diagram of a traditional RF-sampling receiver; (b) Traditional RF-sampling receiver using a time delay $\Delta t$ for quadrature demodulation.

scaling [3]-[6], [23] and system-on-chip (SoC) integration [4], [5], for instance, by extensively using of switches, capacitors and timing. Switching speed is a MOS device performance indicator that steadily improves with technology scaling [6]. The baseband selectivity is digitally controlled by the clock frequency and the capacitance ratio, both of which can be very precise in deep-sub-micron CMOS [3] and therefore be robust to process variation and improve the yield. Migration from one CMOS process node to the next may also be easier [4]. As CMOS continues scaling and the SoC trend goes on, it seems worthwhile to explore the potential of building a SDR receiver based on RF-sampling techniques.

We will now focus on an RF-sampling receiver exploiting the DT-mixing architecture as sketched in Fig. 2(b), and show how it makes RF sampling more suitable to SDR receivers.

\section{Discrete-Time MiXING ReCEIVER ARChitecture}

Fig. 3(a) shows a traditional RF-sampling receiver, consisting of an RF pre-select filter, LNA, RF sampler, a chain of SC-circuits for filtering and decimation, IF amplifier (IFA), and ADC. However, the traditional RF-sampling receivers face a few challenges when applied to SDR, including 1) the narrowband quadrature demodulation and 2) the noise and interference folding due to aliasing. We will propose an architecture-level solution, namely DT mixing, which allows both wideband quadrature demodulation and wideband harmonic rejection to reduce aliasing. This differs from traditional RF-sampling receivers, which realize downconversion via CT-to-DT sampling or DT re-sampling. Next, we will address the two challenges mentioned above.

\section{A. DT Mixing Concept and Wideband Quadrature Demodulation}

Known RF-sampling receivers often use "second-order sampling" [24]: a time delay $\Delta t$ between the two sampling paths approximates a desired phase shift, e.g., $90^{\circ}$ for I/Q demodulation, as shown in Fig. 3(b). The resulted phase shift $\Delta \varphi$ between I and $Q$ at IF is given by $[12, \text { Section III }]^{5}$

$$
\Delta \varphi=2 \pi \cdot f_{\mathrm{RF}} \cdot \Delta t
$$

where $f_{\mathrm{RF}}$ is the frequency of the RF input signal (not the center frequency). Thus, the desired exact $\Delta \varphi$ is only obtained at specific RF-frequencies, e.g., for $90^{\circ}$ only frequencies of $\{(k+$ $0.25) / \Delta t\}$ where $k$ is an integer.

Usually $\Delta t$ is chosen in such a way that the phase shift is exact for the center frequency $\left(f_{c}\right)$ of the desired RF signal. Rearranging (4), and substituting $\Delta \varphi=\pi / 2+k \cdot 2 \pi$, the following condition holds:

$$
\Delta t=\frac{\pi / 2+k \cdot 2 \pi}{2 \pi f_{c}}=\frac{k+0.25}{f_{c}} .
$$

The absolute phase error at (arbitrary) frequency $f_{\mathrm{RF}}$ then is

$$
\left|\varphi_{e}\right|=\left|2 \pi \cdot\left(f_{\mathrm{RF}}-f_{c}\right) \cdot \Delta t\right|
$$

In contrast, the phase shift generated by a CT-mixing receiver can be written as $\Delta \varphi=2 \pi \cdot f_{\mathrm{LO}} \cdot \Delta t$, which is proportional to the LO frequency $\left(f_{\mathrm{LO}}\right)$ instead of $f_{\mathrm{RF}}$. If $\Delta t=1 /\left(4 f_{\mathrm{LO}}\right), \Delta \varphi$ can be constantly $90^{\circ}$ over any $f_{\mathrm{RF}}$. Hence, there is no systematic $\mathrm{I} / \mathrm{Q}$ phase error for CT-mixing.

Larger phase error leads to less accurate quadrature demodulation and degraded image rejection. From (6), we can see the maximum phase error is proportional to the channel $\mathrm{BW}$ which is $2 \cdot\left|f_{\mathrm{RF}, \max }-f_{c}\right|$ for a zero-IF receiver, and the time delay $\Delta t$ which is inversely proportional to $f_{c}$ according to (5). Note that the sampling frequency $\left(f_{s}\right)$ does not determine the phase error. For the smallest $\left|\varphi_{e}\right|$, we need the smallest time delay, i.e.,

\footnotetext{
${ }^{5}$ To assist the understanding, here we correct two printing errors during the PDF conversion in [12]: a) just above Fig. 2 of [12], " $0 \cdot \Delta t<T_{s}$ " should be " $0 \leq \Delta t<T_{s}$ "; b) in the third line below Fig. 3 of [12], " $\left(t-n T_{s}-\Delta t\right)$ " should be " $\Sigma \delta\left(t-n \cdot T_{s}-\Delta t\right)$ ".
} 


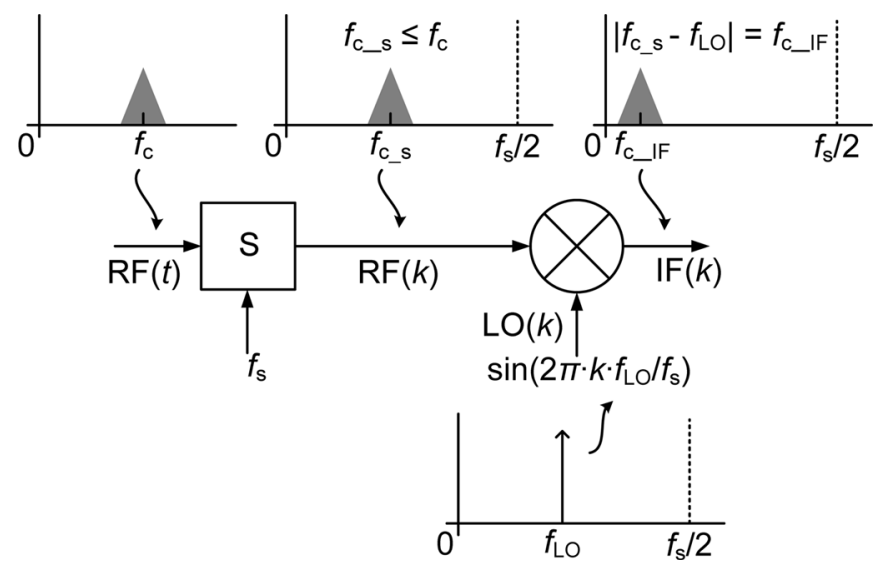

Fig. 4. A basic discrete-time mixing downconverter.

$k=0$ so $\Delta t=0.25 / f_{c}$. At GHz frequencies $\left(f_{c}\right)$ and a few $\mathrm{MHz}$ channel BW, the error can still be acceptable without correction, but for lower $f_{c}$ or larger channel $\mathrm{BW}$ it easily becomes several degrees. For example, for ultra-wideband applications, a $528 \mathrm{MHz}$ channel around a $3.432 \mathrm{GHz}$ carrier in a zero-IF receiver leads to $f_{c}=3.432 \mathrm{GHz}$ and $\left|f_{\mathrm{RF}, \max }-f_{c}\right|=264 \mathrm{MHz}$. According to (6) considering $\Delta t=0.25 / f_{c}$, it means a maximum phase error of $7^{\circ}$. For SDR applications which are aimed to accommodate any standards, such phase error is undesired.

Since the phase error can be predicted as (6), it should be possible to compensate it in the digital domain [25] at the cost of extra power and complexity. On the other hand, the phase shift introduced by mixing is theoretically constant over frequency since the multiplication operation conveys the phase from the LO to the IF signal. We propose to retain this favorable property of mixing in an RF-sampling receiver, via a DT-mixing architecture.

The basic DT-mixing downconverter is shown in Fig. 4, consisting of a sampler followed by a DT mixer. Now we explain its general working principle. The sampler running at $f_{s}$ converts the RF input signal centered at $f_{c}$ from $\mathrm{CT}$ to DT domain, i.e., $R F(t)$ to $R F(k)$. In principle, there is no specific requirement on the ratio of $f_{s} / f_{c}$, i.e., subsampling, Nyquist sampling and oversampling are all possible. Assume the sampled signal $\mathrm{RF}(\mathrm{k})$ has a center frequency of $f_{c_{-} s}$ and is sampled at a rate of $f_{s}$, where $f_{c_{-} s} \leq f_{c}$ holds as a result of sampling. In the DT mixer the signal $\mathrm{RF}(\mathrm{k})$ is mixed with a DT sine-wave $\mathrm{LO}(\mathrm{k})$ of frequency $f_{\mathrm{LO}}$ and also sampled at $f_{s}$. The IF output signal IF(k) is centered at $f_{c_{\perp} \mathrm{IF}}$ (see Fig. 4) and the relationship $f_{c_{-} \mathrm{IF}}=\left|f_{c_{-} s}-f_{\mathrm{LO}}\right|$ holds as a result of mixing downconversion. Note that downconversion may happen in both the sampler and the DT mixer stages.

For I/Q DT mixing, as shown in Fig. 5, a single sampling stage converts a CT signal $\mathrm{RF}(\mathrm{t})$ into the DT domain, followed by two DT mixers multiplying the sampled signal $\mathrm{RF}(\mathrm{k})$ with a DT cosine-wave $\mathrm{LO}_{I}(k)$ and a DT sine-wave $\mathrm{LO}_{Q}(k)$ respectively. For zero-IF downconversion, the DT cosine or sine frequency $f_{\mathrm{LO}}$ is the same as the sampled signal center frequency $f_{c_{-} s}$, i.e., $f_{\mathrm{LO}}=f_{c_{-} s}$. The DT cosine and sine waves have a $90^{\circ}$ phase difference, transferred to IF via multiplication, similar to what a CT mixer does. Thus, the phase shift via DT mixing is frequency independent.

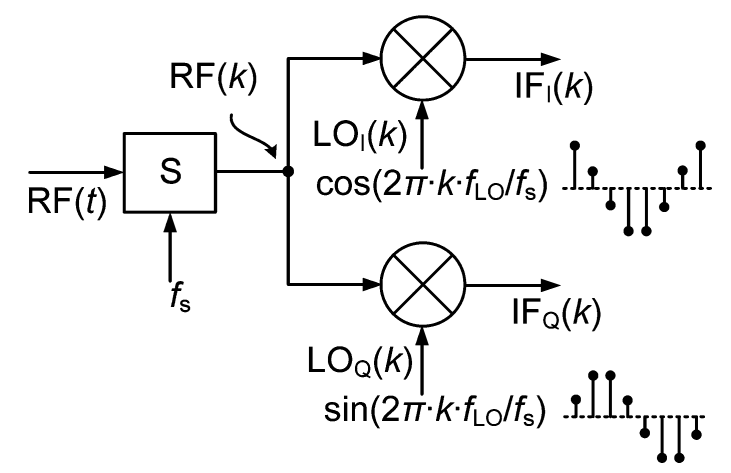

Fig. 5. A discrete-time (DT) mixing downconverter using I/Q DT mixers.

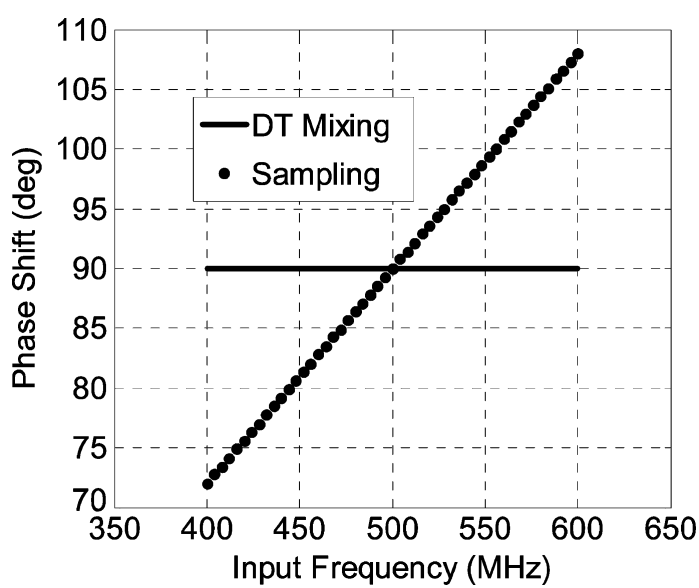

Fig. 6. Simulated phase difference between the I and Q IF-signals as a function of the input RF at a center frequency of $500 \mathrm{MHz}$, showing mixing has benefits in maintaining 90 degrees over a wide band.

We did simulation to prove the different effects on I/Q phase shift by a traditional CT-to-DT sampling technique [Fig. 3(b)] and our proposed DT-mixing technique (Fig. 5). For both cases, we used ideal components, e.g., ideal switches and capacitors. The RF input signal is centered at $500 \mathrm{MHz}$ with a $200 \mathrm{MHz}$ $\mathrm{BW}$ and the output is at zero-IF. The phase difference between the I and Q outputs are plotted in Fig. 6, showing almost a $20^{\circ}$ error for the traditional sampling approach, as predicted by (6), while maintaining wideband constant $90^{\circ}$ for DT mixing.

\section{B. Wideband Harmonic Rejection}

Another challenge of an RF-sampling receiver is aliasing, as noise and interference around harmonics of the sampling frequency are folded to baseband during the sampling process. For narrowband applications, a dedicated RF filter can help [Fig. 3(a)], but for SDR applications such a filter limits the flexibility. Other solutions are desired to at least relax the requirements on the RF filter.

For a CT mixer, it is well known that all even-order harmonics can be rejected by applying differential LO signals. Moreover, in [26], a harmonic-rejection (HR) technique for CT mixers in a transmitter was proposed to suppress some odd-order harmonics. CT HR mixers have been applied for digital TV applications [27], [28]. Using a sum of weighted multi-phase squarewave clocks, an effective LO with reduced harmonic content 
(a)

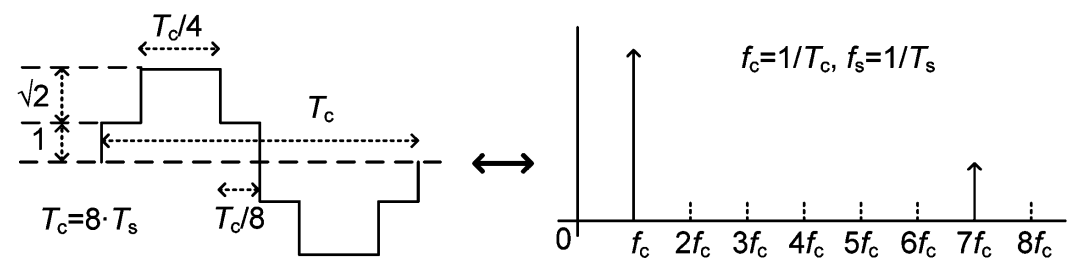

(b)

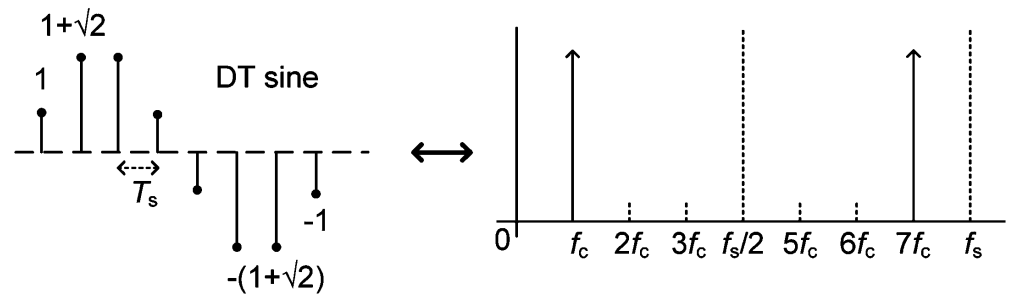

Fig. 7. Effective LO waveform for 2 nd to 6 th harmonic rejection and the corresponding spectrum: (a) continuous-time; (b) discrete-time.

is created (see Fig. 7). In general, the number of rejected harmonics is limited by the number of available clock phases. For example, as shown in Fig. 7(a), an effective LO summed from three $T_{c} / 8$-time-shifted square-waves with an amplitude ratio of $1: \sqrt{ } 2: 1$ is free of the 2 nd to 6th harmonics, and only contains the $(8 n \pm 1)$ th harmonics $(n=0,1,2 \ldots$ so only 1 st, 7 th, 9th, etc.).

A similar idea can be applied to the DT mixers to reject sampling aliases (harmonics). Since the DT sine-wave has sampling aliases located at $\left(n \cdot f_{s} \pm f_{\mathrm{IF}}\right)$, high sample rate reduces the amount of in-band aliases (for a given RF-filter BW). So oversampling $\left(f_{s}>2 f_{\mathrm{RF}}\right)$ is more effective. For zero-IF downconversion, we can choose $f_{s}=m \cdot f_{c}(m=1,2,3 \ldots)$. The remaining harmonics causing aliasing are the $(m \cdot n \pm 1)$ th, and the first un-wanted harmonic is the $(m-1)$ th. The further-away the first unwanted harmonic, i.e., a larger $\mathrm{m}$, the less requirements on the RF filter. Fig. 7(b) shows an example of the DT sine-wave with $m=8$, corresponding to Fig. 7(a).

A FIR filter in the signal path can in principle also reject sampling aliases by positioning the filter notches at the targeted antialias frequencies [29]. However, it is only effective for a limited channel BW due to the limited notch BW intrinsic to any FIR filter. Alternatively, the stop-band of a FIR filter could also reduce harmonic interferers, where the rejection bandwidth is limited by the pass-band and the stop-band frequencies. To achieve relatively wideband rejection and a good rejection ratio, the required filter order and filter coefficients are often too complicated to be practical for an analog-DT implementation.

For SDR applications, any useful channel BW should be accommodated, so a good HR ratio over a wide channel BW is important. Wideband HR is also important to reduce the co-channel distortion caused by strong wideband interferers around harmonic frequencies even if these interferers do not directly overlap with the desired channel at baseband. Therefore, a FIR-filter based HR technique does not suit SDR applications. In contrast, the DT-mixing based HR technique does not have intrinsic channel BW limitations, i.e., it presents wideband $\mathrm{HR}$, as for a $\mathrm{CT}$ mixer.

Besides improving signal-to-interference ratio, oversampling and harmonic rejection in a sampling receiver will also improve signal-to-noise ratio (or equivalently NF) since the noise folding is also reduced, in the same way as rejecting interference.

Summarizing, we proposed an alternative receiver architecture using RF sampling followed by DT mixing with a sampled cosine or sine wave. It enables wideband quadrature demodulation and wideband harmonic rejection, making RF sampling more suitable for SDR receivers.

\section{PROOF-OF-CONCEPT IMPLEMENTATION}

To prove the concept of DT mixing, we implemented a prototype receiver to cover 0.2 to $0.9 \mathrm{GHz} \mathrm{RF}$ range, targeting the CR applications recently proposed in the television broadcasting band [14]. The wideband features of DT mixing can be useful for CR applications which might use non-contiguous segments of free spectrum distributed over a wide band.

\section{A. Receiver Architecture}

Fig. 8 shows the architecture of the receiver. For clarity it is shown single-ended, but in fact all circuits are implemented differentially. Please note that the LNA is not included in this work, but is included in [15]. An inverter-based RF voltage amplifier (RFA) delivering $6 \mathrm{~dB}$ gain drives a passive switched-capacitor (SC) core consisting of three stages. The first stage is effectively an oversampler (S), with a sample rate 8 times the input center frequency, i.e., $f_{s}=8 f_{c}$, for 2 nd to 6 th harmonic rejection [Fig. 7(b)]. The second stage consists of I/Q DT mixers for downconversion to zero-IF $\left(f_{\mathrm{LO}}=f_{c}\right)$. The third stage is a low-pass IIR filter (LP IIR), removing undesired interference and serving as an antialiasing filter before decimation to lower sample rate. For measurement purpose, the quadrature IF outputs are buffered via source-followers (B) with a voltage gain of 1. An on-chip clock generator, consisting of clock buffer (CB), divide-by-4, and 1/8-duty-cycle driver, controls the SC core.

Fig. 9 illustrates how this DT HR mixer works. The I/Q DT mixers shown in Fig. 8 multiply the incoming samples with DT cosine and sine waves, i.e., weighting ratio of $1:(1+\sqrt{ } 2)$ if cosine and sine waves with frequency $f_{c}$ are sampled at $8 f_{c}$. Since the DT clock is periodic with $f_{\mathrm{LO}}=f_{c}$, its spectrum only contains an impulse at $f_{c}$ within the DC to $f_{s} / 2$ range. Multiplying the oversampled signal with the DT clock will down-convert 


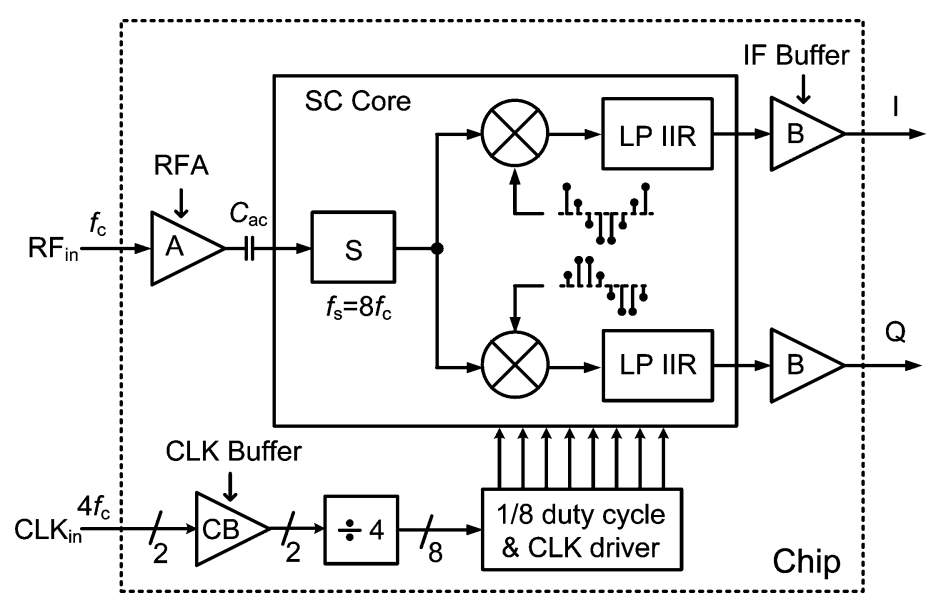

Fig. 8. Block diagram of the implemented DT-mixing receiver with 8 times oversampling and 2nd-to-6th harmonic rejection.

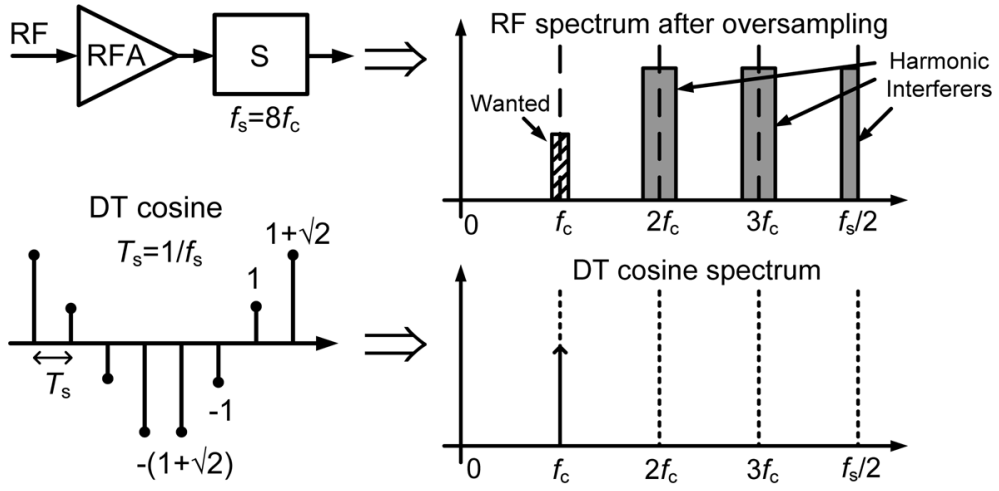

IF spectrum after DT harmonic-rejection mixing

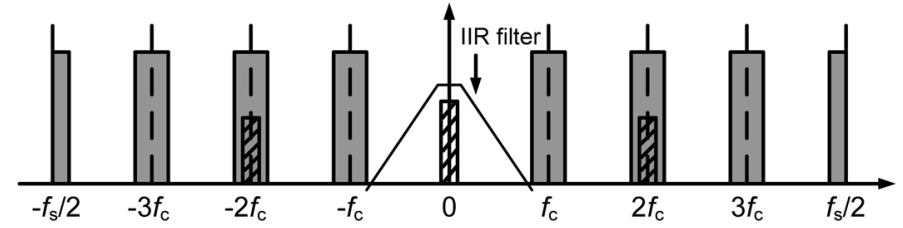

Fig. 9. Mechanism of the discrete-time (DT) harmonic-rejection mixing.

the signal from $f_{c}$ to DC without folding harmonics at $2 f_{c}, 3 f_{c}$, and $4 f_{c}$. However, the harmonics already folded to $f_{c}$ during the sampling process cannot be differentiated from the wanted signal. Since $f_{s}=8 f_{c}$, during sampling, the 7 th harmonic folds to $f_{c}$, and the 5 th harmonic folds to $3 f_{c}$, etc. Therefore, the un-suppressed RF harmonics are the 7th, 9th, 15th, 17th etc, but the close-by 3rd and 5th and all even-order harmonics are removed. Mixing with the DT cosine and sine waves also leads to a true frequency-independent $90^{\circ}$ phase shift for wideband quadrature demodulation.

To better understand the implementation, a more detailed description for some of the key blocks will follow.

\section{B. Circuit Implementation}

1) RFA: The RFA is shown in Fig. 10, which is single-ended and consists of two inverters. Two copies of such an RFA are employed for differential operation. For a gain of two, the driving inverter, with a large feedback resistor for automatic DC bias, is twice the size of the loading inverter, with a small feedback resistor $R_{f b}$ around $2 /\left(g_{m, P}+g_{m, N}\right)$ to partially compensate

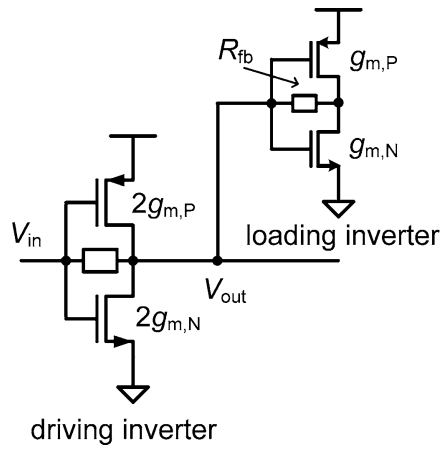

Fig. 10. The schematic of RFA based on inverters.

the 3rd-order nonlinearity [15]. Nominally, the driving inverter draws $1 \mathrm{~mA}$ with a total $g_{m}$ of $20 \mathrm{mS}$ and the loading inverter takes $0.5 \mathrm{~mA}$ with $10 \mathrm{mS}$.

Please note that the RFA is not a complete LNA and its input is not matched to $50 \Omega$ but targeted at high impedance. Functionally the RFA is not required for realizing the DT mixing 


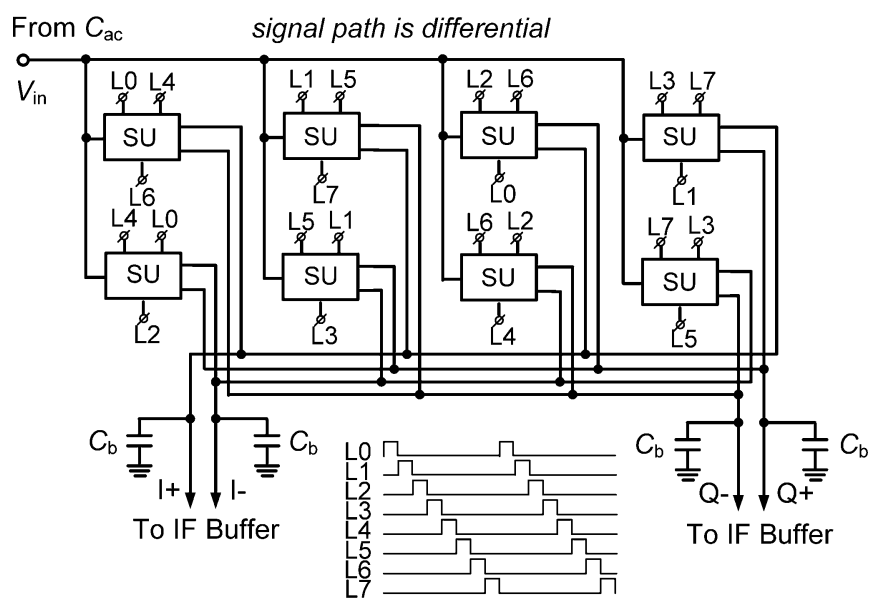

(a)

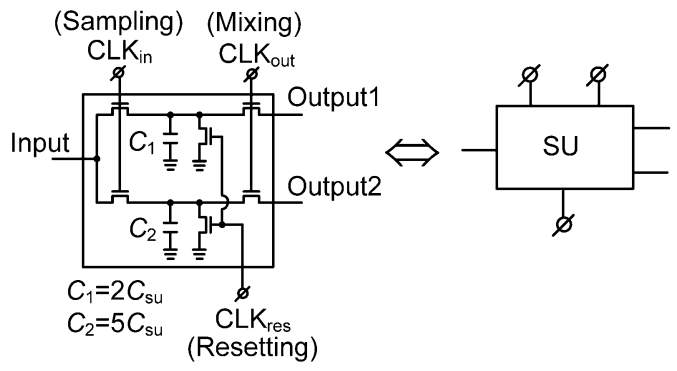

(b)

Fig. 11. (a) Switched-capacitor (SC) core circuitry with 8-phase clock waveform. (b) Transistor-level construction of a sampling unit (SU).

concept. It mainly serves as a buffer to drive the follow-up SC circuitry for measurement purposes. Without a buffer, the input impedance of a SC chip is more complicated, which can depend on the driving source impedance [19].

2) SC Core: Fig. 11(a) shows the SC core circuitry. In total, there are 16 sampling units (SU) implemented, but for clarity only half of the differential system is shown. An AC coupling capacitor $\left(C_{a c}=2.5 \mathrm{pF}\right)$ is used between RFA and the SC core input as shown in Fig. 8, so that the sampling units can work at a common-mode (CM) level of ground (GND), which is defined by reset switches inside the sampling units [see Fig. 11(b)]. The clock (L0-L7) swings from GND to VDD to minimize the switch-on resistance of nMOS switches, for a good linearity and a small time constant. The clock design also aims at a fast falling edge to reduce distortion introduced at the sampling moment. Four buffer capacitors $C_{b}$ (18 $\mathrm{pF}$ each) are used to store the signal charge for quadrature outputs, built via nMOS accumulation capacitors for small area.

Fig. 11(b) shows a sampling unit in detail. In each SU, there are two weighted sampling capacitors to map the $1:(1+\sqrt{ } 2)$ weighted pulse amplitudes needed for a DT cosine or sine wave. In previous work, weighted amplifiers were used [9], [26]-[29]. We exploit weighted capacitors $\left(C_{1}, C_{2}\right)$ which can have superior matching properties for good amplitude (weighting factor) accuracy in HR. To make a non-integer $1:(1+\sqrt{ } 2)$ ratio reliable in layout is difficult. We used unit capacitors with value $C_{s u}$ in a 2:5 ratio as approximation $\left(C_{1}=2 C_{s u}=160 \mathrm{fF}\right.$, $C_{2}=5 C_{s u}=400 \mathrm{fF}$ ), built via metal-oxide-metal (MOM) capacitors for high linearity. In each SU, there are three pairs of switches controlled by three different clock phases, and in each pair the two switches also share the same weighting factor as the two capacitors. The switch pair for sampling ("sampling switches") is controlled by $\mathrm{CLK}_{\mathrm{in}}$, and the switch pair for DT mixing ("mixing switches") is controlled by CLK out $_{\text {. The third }}$ switch pair for resetting ("resetting switches") controlled by $\mathrm{CLK}_{\text {res }}$ defines the CM level of all switches to GND.

The working procedure of a SU is as follows, taking the upper left unit in Fig. 11(a) as an example:

1) at clock phase L0 (CLK $\mathrm{Cn}_{\text {in }}$ high), the sampling switches take the same RF voltage to two sampling capacitors $C_{1}$ and $C_{2}$;

2) then the charge will be kept for three idle phases, and at

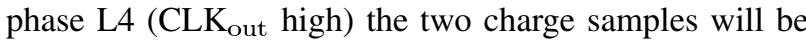
transferred via the "mixing switches" to two buffer capacitors $C_{b}$, one in I path (I+ side) and the other in Q path (Qside);

3) waiting for another idle phase, in phase L6 (CLK res $_{\text {high) }}$ the reset switches clean up the sampling capacitors $C_{1}$ and $\mathrm{C}_{2}$;

4) and after one more idle phase, the whole procedure repeats from phase LO.

The other 7 units experience the same pattern of activity but shifted in time. By inserting some idle phases between each operation (sampling, mixing, or resetting), we can guarantee the three switching operations do not disturb each other during the phase transitions.

Next we will describe each functional blocks, i.e., the sampler, the DT mixer, and the IIR filter, in the SC core of Fig. 8.

a) Sampler: We aim for a voltage sampler, to reduce the frequency dependency of conversion gain [12], although it suffers from noise folding. Oversampling can reduce the noise folding and hence improve NF. The oversampling function is implemented via a time-interleaved sampling structure. Eight interleaved sampling units are controlled by 8-phase non-overlapping clocks with $1 / 8$ duty cycle, as shown in Fig. 11(a). Each of the 8-phase clocks gives a sample rate of $f_{c}$, so 8-times interleaving results in an effective sample rate of $8 f_{c}$. Using time-interleaved sampling to achieve oversampling, there is no extra cost on clock speed compared to other HR systems [9], [26]-[29], where multiphase clocks are also employed. Note that achieving 8-times oversampling only requires doubled clock speed compared to what is needed for regular quadrature downconversion, where 4-phase clock is used.

The switch size is selected so that the switch-on resistor and the sampling capacitor forms a relatively small time constant $\tau=20$ ps. Since the targeted upper input center frequency $\left(f_{c}\right)$ is $900 \mathrm{MHz}$ and the clock duty cycle is $1 / 8$, the minimum switch-on time per period is $139 \mathrm{ps,} \mathrm{which} \mathrm{corresponds} \mathrm{to} 7 \tau$ and guarantees small voltage signal attenuation [30].

b) DT Mixer: The DT mixing is implemented in the charge domain, via a systematic combination of the mixing switches from all SUs to transfer the charge samples from the sampling capacitors $\left(C_{1}, C_{2}\right)$ to the buffer capacitors $\left(C_{b}\right)$. Effectively the DT mixing is realized via de-multiplexing as shown in Fig. 12. The oversampled charge data stream $\mathrm{RF}(\mathrm{k})$ goes through the switching network controlled by $\mathrm{CLK}_{\text {out }}$ in Fig. 11(b) and becomes IF(k). For illustration, Fig. 12 actually 


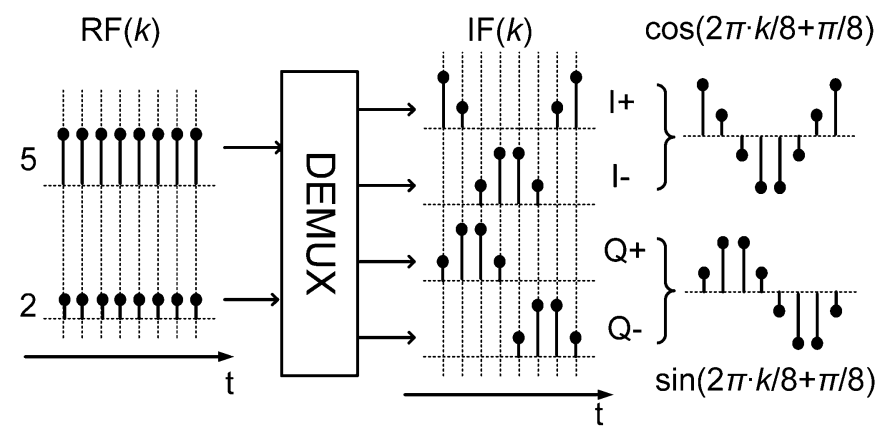

Fig. 12. To implement discrete-time mixing via de-multiplexing.

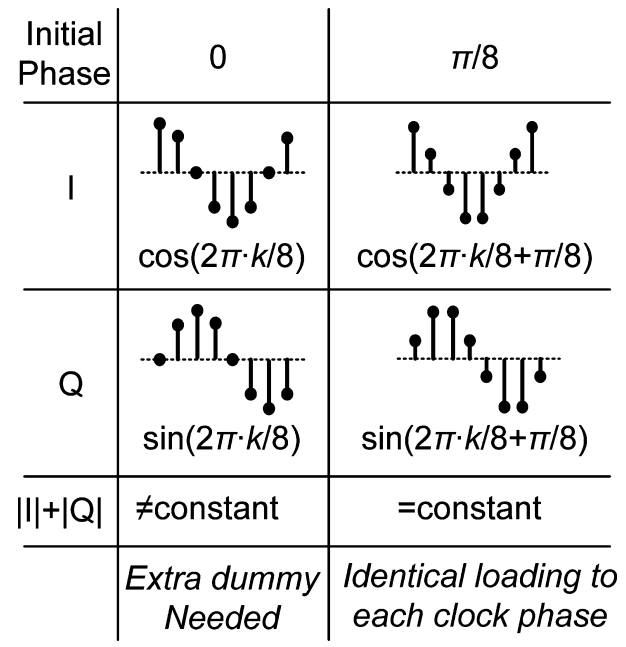

Fig. 13. Comparison of two initial phase choices for the DT cosine and sine: $\pi / 8$ is preferred.

shows an example with a $\mathrm{DC}$ input $\mathrm{RF}(\mathrm{k})$ which is up-converted to become a quadrature DT sinusoidal output $\mathrm{IF}(\mathrm{k})$. But the same principle applies to downconversion. Note that the DT-cosine or DT-sine also has a sample rate of $8 f_{c}$ but the frequency of the DT cosine-wave or DT sine-wave is $f_{c}$, as illustrated in Figs. 7(b) and 9.

Changing the initial phase of the DT cosine and sine, the resulting $\mathrm{LO}$ waveform can be different, leading to a different ratio among sampling capacitors. As shown in Fig. 13, by choosing the initial phase to be $\pi / 8$, we have

$|\cos (2 \pi \cdot k / 8+\pi / 8)|+|\sin (2 \pi \cdot k / 8+\pi / 8)|=$ constant.

This choice guarantees the identical loading for all clock phases without extra dummy needed. The identical loading for each clock phase is important for both gain and phase accuracies.

Conversion gain (CG) is an important parameter of mixer. In general, the systematic CG of a DT mixer can be derived [31] as

$$
C G=\frac{1}{2}\left(\sum_{i=1}^{n}\left|p_{i}\right| / n\right)^{-1} .
$$

In (8), the " $n$ " is the ratio of the sampling frequency to the DT sine-wave frequency $\left(n=f_{s} / f_{\mathrm{LO}}\right)$, so that $n / 2$ is the oversampling ratio. In another way of interpreting, the " $n$ " is the total number of discrete time points in one period of the DT sine-wave, and each discrete time point has a weighting factor for its relative amplitude. The " $p_{i}$ " is the value of one of the weighting factors in the DT sine-wave $(i=1,2, \ldots, n)$. There is a " $1 / 2$ " factor in (8) since the output signal amplitude at the difference frequency is halved compared to the input, just like in a CT mixing. The periodic DT sine-wave can be described by a vector $p=\left[p_{1}, p_{2}, \ldots, p_{n}\right]$. For example, in Fig. 13, for the DT sine-wave with an initial phase of $\pi / 8$, its $n=8$ and its $p_{i}=\sin [2 \pi \cdot(i-1) / 8+\pi / 8]$, i.e., $p=[0.38,0.92,0.92,0.38,-0.38,-0.92,-0.92,-0.38]$. According to (8), the CG for such a DT mixer is 0.77 .

To verify the systematic CG, we rebuild and simulate the schematic of Fig. 11(a) using ideal switches and capacitors to eliminate parasitic effects which are not considered in (8) and without applying RFA and $C_{a c}$. The result of $-2.3 \mathrm{~dB}$ fits exactly what we derived above, i.e., 0.77. The overall gain of the receiver should consider the cascaded gain of RFA $(6 \mathrm{~dB})$, voltage sampler $(0 \mathrm{~dB})$, DT mixer $(-2.3 \mathrm{~dB})$, IIR filter $(0 \mathrm{~dB})$, and IF buffer $(0 \mathrm{~dB})$, which is then equal to $6-2.3=3.7 \mathrm{~dB}$, without considering 2 nd-order effects such as parasitic capacitance.

c) IIR Filter: The charge sharing operation, between the sampling and the buffer capacitors, implements a low-pass IIR filter [3], [23]. The voltage transfer function of this IIR filter can be written as

$$
\begin{aligned}
& H_{I I R}(Z)=\frac{1-\beta}{1-\beta \cdot Z^{-1}} ; \\
& \left\{\beta \approx \frac{1}{2}\left(\frac{C_{b}}{C_{b}+C_{1}}+\frac{C_{b}}{C_{b}+C_{2}}\right)\right\} .
\end{aligned}
$$

According to (9), the IIR filter has a voltage gain of 1 at DC. The effective factor $\beta$ is an average of these two charge transfer functions $F_{1}=C_{b} /\left(C_{b}+C_{1}\right)$ and $F_{2}=C_{b} /\left(C_{b}+C_{2}\right)$. Equivalently, $\beta$ can be written as $C_{b} /\left(C_{b}+C_{I I R}\right)$ and the IIR filter sees an effective sampling capacitor $C_{I I R} \approx\left(C_{1}+C_{2}\right) / 2$, if $C_{1} \ll C_{b}$ and $C_{2} \ll C_{b}$.

The IIR filter BW is determined by the sample rate $f_{s}$ and the coefficient $\beta$. The clock frequency can be very accurate and therefore $f_{s}$ can be accurate. As seen from $\beta$, the $\mathrm{BW}$ is defined by the capacitor-ratios which can be robust to process variation if the same type of capacitor is used. The matching between $C_{b}$ and $C_{1}$ (or $C_{2}$ ) affects the IIR filter BW. However, since the IIR filter is not the focus of this work, a MOS accumulation capacitor was chosen for $C_{b}$ to save chip area.

As an intrinsic property of DT filters, its BW scales with the sample rate $f_{s}$. To accommodate the 5 to $8 \mathrm{MHz}$ channel spacing used in the television band for the CR applications [14], the $-3 \mathrm{~dB} \mathrm{BW}$ of this IIR filter is designed to be roughly $10 \mathrm{MHz}$ when $f_{c}=0.5 \mathrm{GHz}$ (or equivalently $f_{s}=4 \mathrm{GHz}$ ), which is around the middle of the $0.2 \mathrm{G}-0.9 \mathrm{GHz}$ band. In simulation, the $-3 \mathrm{~dB}$ BW is $4.4 \mathrm{MHz}$ at $0.2 \mathrm{GHz} \mathrm{LO}$ and $16.9 \mathrm{MHz}$ at $0.9 \mathrm{GHz}$ LO. Normally an IF filter BW that scales with $f_{s}$ is undesired. But this effect can be compensated via a bank of selectable $C_{b}$ values to adapt $\beta$ to different $f_{s}$ [4], [6].

A coarse noise analysis for the IIR filter can be made for Fig. 11(b), viewing the combination of the sampling switch, the sampling capacitor $\left(C_{1}\right.$ or $\left.C_{2}\right)$, and the mixing switch as a 


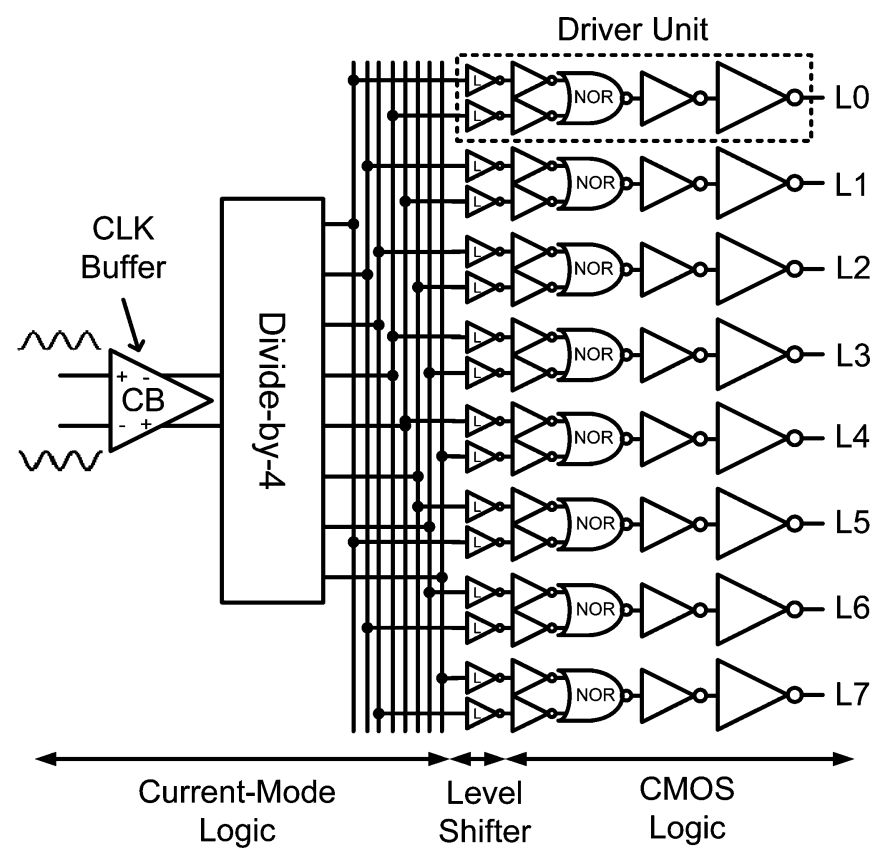

Fig. 14. Block diagram of the 8-phase clock generator.

switched-capacitor resistor, $R_{s c}$. Then the IIR filter can be seen as a low-pass filter formed by $R_{s c}$ and $C_{b}$. The total integrated noise $\left(V_{n}^{2}\right)$ after IIR filtering is $\mathrm{KT} / \mathrm{C}_{b}$. To improve the noise at constant filter BW, both $C_{b}$ and $C_{1} \& C_{2}$ should be scaled up simultaneously.

Besides implementing the IIR filter, the charge sharing operation also shifts the amplitude ratio away from $C_{1}: C_{2}=2: 5$, depending on the charge transfer functions $F_{1}$ and $F_{2}$. Considering $C_{b}=18 \mathrm{pF}, C_{1}=160 \mathrm{fF}$, and $C_{2}=400 \mathrm{fF}$, the effective amplitude ratio is

$$
\begin{aligned}
& \left(C_{1} \cdot F_{1}\right):\left(C_{2} \cdot F_{2}\right) \\
& \quad=\left(C_{1} \cdot \frac{C_{b}}{C_{b}+C_{1}}\right):\left(C_{2} \cdot \frac{C_{b}}{C_{b}+C_{2}}\right) \approx 1: 2.467 .
\end{aligned}
$$

Compared to the desired $1:(1+\sqrt{ } 2)$ ratio, the gain error is reduced from $3.6 \%$ (2:5 ratio) to $2.2 \%$ (1:2.467).

To reduce the sample rate operated by ADC, the outputs of IIR filter can be decimated, e.g., via a moving average, to a lower sample rate and the next stages can use further DT signal processing, e.g., FIR/IIR filter and decimation [3]-[6]. The ADC can work in DT domain, e.g., a DT $\Sigma \Delta$ ADC was used for Bluetooth [3] or for GSM [32] and a DT successive-approximation ADC for Wi-Fi/WiMax [6]. Alternatively, the ADC can also work in CT domain when the baseband sample rate is high enough and the CT filters can remove high-frequency images, e.g., a CT $\Sigma \Delta$ ADC for GSM/GPRS [4].

3) 8-Phase Clock Generator: We need 8-phase clocks to drive the SC core described above. Such a clock generator is shown in Fig. 14. It can be partitioned into three parts, namely a high-frequency current-mode logic (CML) part, a level shifter, and a CMOS logic part. The CMOS logic works with full-swing signals while the CML does not, and the level shifter bridges the gap.

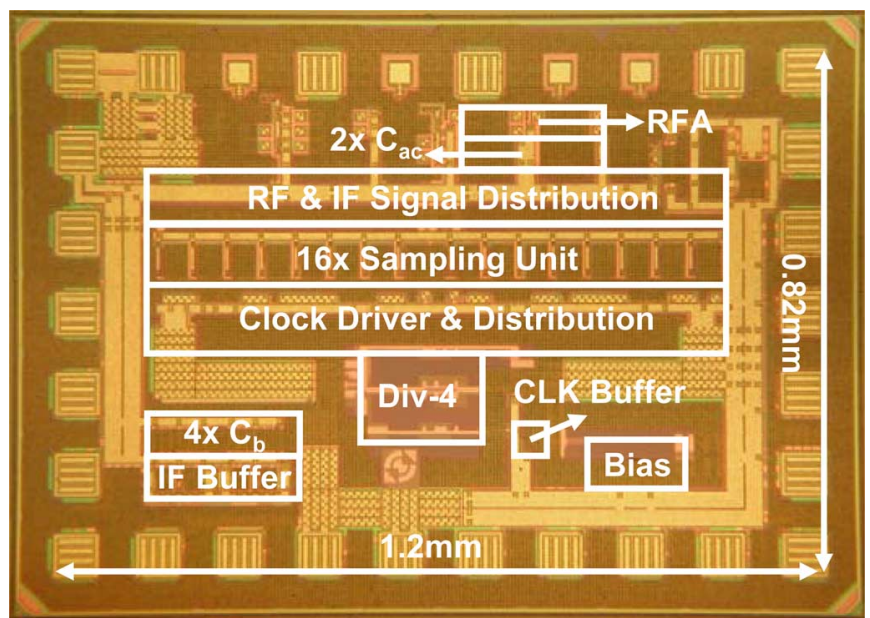

Fig. 15. Microphotograph of the chip fabricated in $65 \mathrm{~nm}$ CMOS with key blocks indicated.

The input receives external differential clocks at frequency $4 f_{c}$. An nMOS CML buffer helps recover the clock steepness and reject possible common-mode interference. This buffer drives a divide-by- 4 circuit consisting of four nMOS CML latches to generate 50\%-duty-cycle 8-phase clocks at frequency $f_{c}$. The CML is chosen for its fully differential operation which has less effect and more immunity to the voltage supply than its CMOS counterpart.

The following stages are split into 8 paths each with a tapered driver unit. Each driver unit consists of level shifters, inverters and NOR gates to generate a 1/8-duty-cycle full-swing clock. The level shifter is simply implemented by an inverter with stronger driving capability in pMOS than in NMOS. CMOS logic is necessary at the final stages since full-swing clocks can improve both noise and linearity of the sampling circuitry by minimizing the switch-on resistance.

\section{EXPERIMENTAL RESULTS}

Fig. 15 shows the microphotograph of the chip fabricated in a baseline $65 \mathrm{~nm}$ CMOS process. The active area of the chip is about $0.4 \mathrm{~mm}^{2}$, most of which is occupied by capacitors $\left(C_{a c}, C_{s u}\right.$ and $\left.C_{b}\right)$ and signal/clock distribution networks. The chip is packaged in a 32-pin Heat-sink Very-thin Quad Flat-pack No-leads (HVQFN) package and measured on PCB. Since the RFA input is the gate of inverter, a $100 \Omega$ SMD resistor for impedance matching is soldered on $\mathrm{PCB}$, close to the package and across the differential RF input traces. Both the receiver inputs and the clock inputs are differential and wideband hybrids (balun) were used to interface to single-ended $50 \Omega$ measurement equipment. The IF-output voltage is sensed by a differential active probe that performs differential to single-ended conversion and impedance conversion to $50 \Omega$. The characteristics of all components and cables for testing are de-embedded from the results.

\section{A. Conversion Gain and Noise Figure}

Fig. 16 (left) shows the simulated ${ }^{6}$ and measured conversion gain (CG) over the 0.2 to $0.9 \mathrm{GHz} \mathrm{RF}$ band. The results are obtained at $1 \mathrm{MHz}$ IF, well within the $-3 \mathrm{~dB}$ IF BW for the whole 

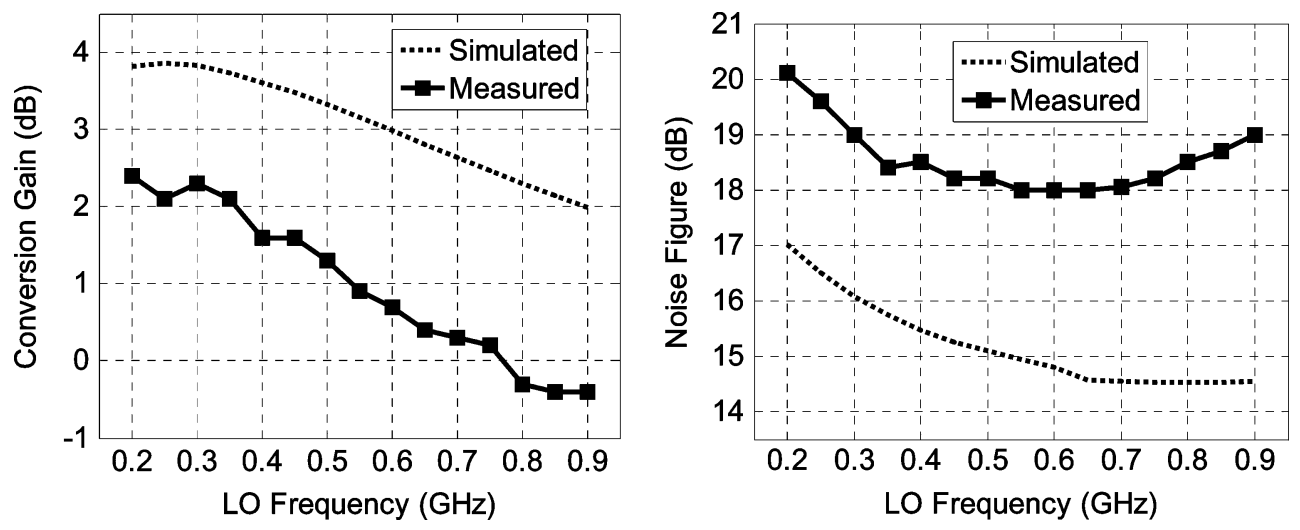

Fig. 16. Simulated and measured (on PCB) conversion gain and DSB NF versus LO frequency (IF $=1 \mathrm{MHz}$ ).

band. The trend of the measured gain fits well with the simulated gain. The CG drops by about $3 \mathrm{~dB}$ from 0.2 to $0.9 \mathrm{GHz}$, indicating the $-3 \mathrm{~dB}$ RF BW. Analyses and simulations indicate that this strong low-pass filtering behavior is mainly due to the parasitic capacitance associated with the AC coupling capacitor $C_{a c}$. To improve the RF BW, the AC coupling capacitor can be moved from the signal path to the clock path, as done in [11]. In layout, long interconnecting wires between the $\mathrm{AC}$ coupling capacitor $C_{a c}$ and the sampling units, as shown in Fig. 15 (RF Signal Distribution), introduce extra parasitic resistance and capacitance. Also the loss of a few centimeter PCB traces for the $\mathrm{RF}$ inputs was not de-embedded. These are the major causes of the gap between the simulated and measured CG.

Fig. 16 (right) presents the simulated and measured DSB NF, both referred to the matched $50 \Omega$ source noise. Two curves show similar trend but the measured NF is about $3-4 \mathrm{~dB}$ worse, due to the degraded gain and the extra noise contributed by the wiring resistance for RF signal distribution (Fig. 15). Simulation shows that the SC-core contributes most noise and the additional $\mathrm{NF}$ contribution from the RFA is less than $1 \mathrm{~dB}$. Theoretically larger gain should bring lower NF, but Fig. 16 shows an opposite trend. In fact, more noise folding at a lower sampling frequency $\left(f_{s}\right)$ raises the NF, even with a higher gain, because: 1$)$ at lower $f_{s}$ the folding of switch noise is more, assuming a fixed $R C$ time constant; 2) at lower $f_{s}$ the folding of source noise and RFA noise is more due to the limited RF BW as indicated by the CG. Simulation shows the RFA 1/f noise has a negligible effect to the NF arise at low band. The measured NF also rises when $\mathrm{RF}$ is close to the upper-side limit, where the clock swing is insufficient to fully turn on the switches. As a result, less signal can pass through to IF and also the switches become noisier, so SNR is degraded at the output.

The CG and DSB NF in $[13]^{7}$ were measured on wafer via probing, which showed a different trend. It's very likely due to the different way of installing the off-chip matching resistors. During wafer probing, two $50 \Omega$ resistors for input matching were screwed on top of the probe and thus these $50 \Omega$ resistors are further away from the chip $(\sim 4 \mathrm{~cm})$ and this will introduce a transmission-line effect leading to the trend shown in [13],

\footnotetext{
${ }^{6}$ All simulation results shown in this paper were carried out on schematic level, and no wire parasitics were included.

${ }^{7}$ The NF shown in [13] should be DSB NF instead of SSB NF.
}

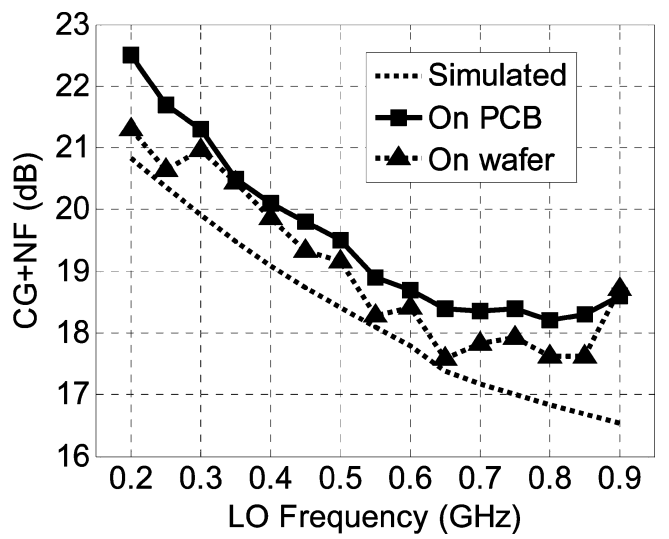

Fig. 17. Normalized output noise versus LO frequency, calculated by summing the conversion gain $(\mathrm{CG})$ and noise figure $(\mathrm{NF})$ : simulation and measurements based on PCB and wafer probing fit better.

as being verified by simulation. Nevertheless, since the noise from the chip is much larger than the noise from the source as indicated by the large NF, we may compare the normalized output noise (Fig. 17), via the sum of CG and NF in $\mathrm{dB}$, which can get rid of the transmission-line effect and indicates mainly the circuit-generated noise at its output. Then three cases, i.e., simulated, measured on PCB and measured on wafer, show a similar trend and their discrepancy is within $1.5 \mathrm{~dB}$.

\section{B. HR Ratio}

Fig. 18 compares the HR ratio using two different techniques to reject harmonics: DT mixing and conventional FIR filtering [29]. As predicted by theory, the HR ratio of a sampling downconverter using FIR filtering drops significantly over the channel (simulation), while the proposed DT-mixing architecture gives wideband HR without channel BW limitation (both simulation and measurement). For DT mixing, the trend of measured results (one typical sample) fits well with the simulated results. However, phase and amplitude mismatches, which are not included in both simulated curves, degrade the HR ratio in measurement.

Fig. 19 shows the measured HR ratio over the RF band of 10 samples. The worst case 3rd and 5th HR ratios are around $25 \mathrm{~dB}$ and typically they are between $35 \mathrm{~dB}$ and $45 \mathrm{~dB}$. To investigate 


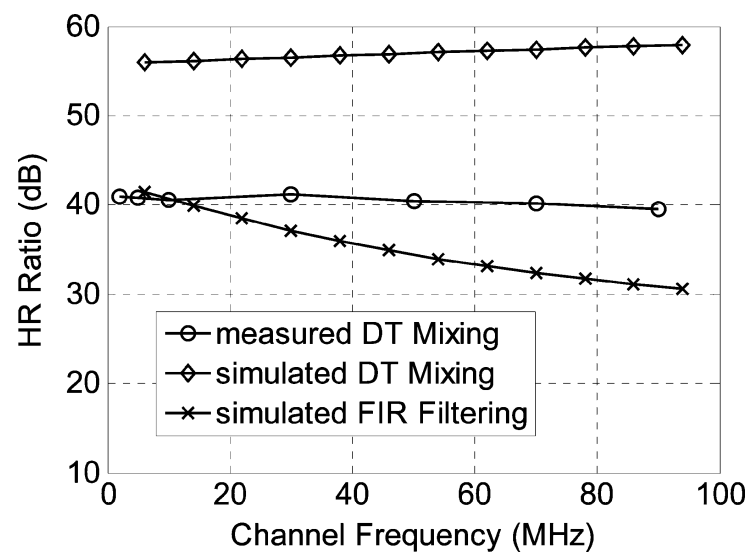

Fig. 18. Simulated (no mismatch) and measured (a typical sample with mismatch) 3rd -order harmonic-rejection (HR) ratio versus channel frequency (LO@0.5 GHz) [measured on wafer].
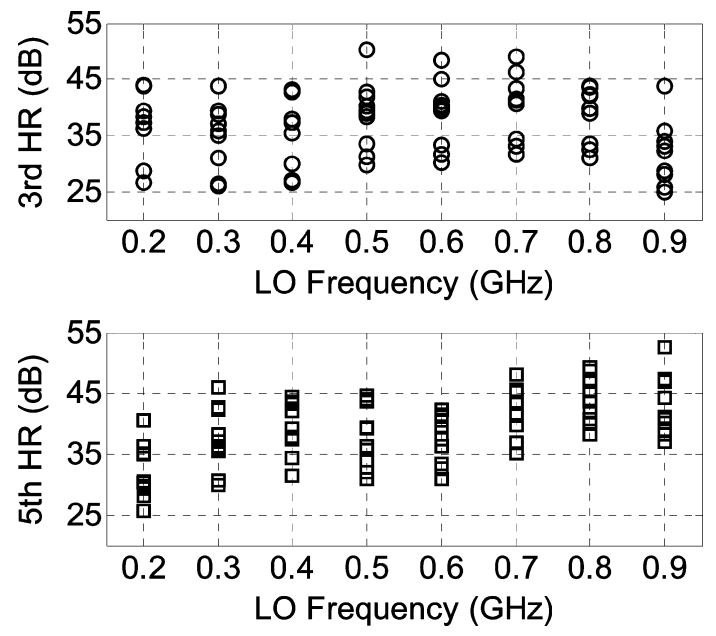

Fig. 19. Measured 3rd and 5th HR ratio versus LO frequency (10 samples) [measured on wafer].

whether phase or amplitude error is dominating, we did Monte Carlo simulations for three configurations: only amplitude error, only phase error, and both. The results are shown in Fig. 20, for 2nd to 6th harmonics of a $0.35 \mathrm{GHz} \mathrm{LO}$. The results indicate that phase error is dominating, which affects not just the 3rd and 5 th HR but also the even-order HR. We also did simulations at $0.7 \mathrm{GHz} \mathrm{LO}$, and the same conclusion holds. The relatively large phase error is mainly due to many buffer stages used after the divider (Fig. 14), leading to large accumulated mismatch. Without using RF filters, the achieved average HR ratio of $40 \mathrm{~dB}$ is comparable to [9], [27], [29] showing only one chip while [28] achieves a minimum $\mathrm{HR}$ ratio of $40 \mathrm{~dB}$. But both amplitude and phase accuracy can still be improved by techniques discussed in [11], which achieve a minimum HR ratio of $60 \mathrm{~dB}$.

The HR ratios are measured with an input power of $-20 \mathrm{dBm}$. When the amplitude of the harmonic interference gets bigger, both amplitude and phase accuracy of the HR mixer can be degraded. The amplitude accuracy is affected because the switch-on resistance is modulated by the instantaneous voltage of the interference. The phase accuracy is affected because the switch-on and switch-off moments are also modulated by the instantaneous voltage of the interference.

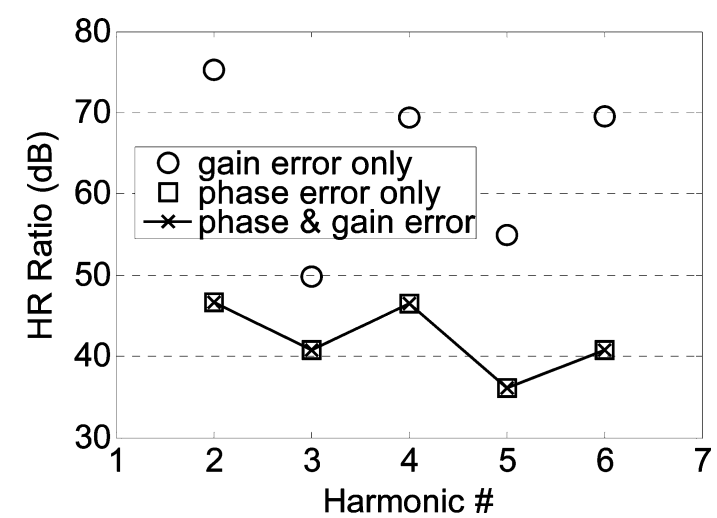

Fig. 20. Simulated 2nd to 6th HR ratio at $0.35 \mathrm{GHz} \mathrm{LO}$ (averaged in $\mathrm{dB}$ for 10 Monte Carlo runs, mismatch only).

TABLE II

SUMMARY OF SOME MEASURED PARAMETERS

\begin{tabular}{|c|c|}
\hline Frequency $(\mathrm{GHz})$ & 0.2 to 0.9 \\
\hline Conv. Gain (dB) & -0.5 to 2.5 \\
\hline DSB NF (dB) & 18 to 20 \\
\hline IIP3 1 & $+10 \mathrm{dBm}$ \\
\hline IIP2 $^{1}$ & $+53 \mathrm{dBm}$ \\
\hline $1 / \mathrm{f} \mathrm{noise} \mathrm{corner}$ & $250 \mathrm{kHz}$ \\
\hline $\begin{array}{c}-3 \mathrm{~dB} \mathrm{IF} \mathrm{BW} \mathrm{@} \\
0.7 \mathrm{GHz} \text { LO }\end{array}$ & $10 \mathrm{MHz}$ \\
\hline VDD & $1.2 \mathrm{~V}$ \\
\hline DC Current & $<16 \mathrm{~mA}$ \\
\hline
\end{tabular}

1 Two tones @ 501M \& 501.4MHz, LO @ 500MHz

\section{Performance Summary}

Table II summarizes some of the measured parameters.

For the measured in-band linearity, the IIP 3 is $+10 \mathrm{dBm}$ and the IIP2 is $+53 \mathrm{dBm}$ with two tones around $500 \mathrm{MHz}$. There are three contributions to distortion: the RFA, the MOSFET switches and the IF buffer. The simulated IIP3 of only the SC core is $+26 \mathrm{dBm}$ and the simulated IIP 3 of only the IF buffer is $+27 \mathrm{dBm}$. This indicates that the measured IIP3 is dominated by the RFA whose linearity can be degraded by process spread and bondwire inductance of VDD/GND supply pins [15]. Since a balun is used in measurement, the IIP3 of each single-ended RFA should be $3 \mathrm{~dB}$ lower, i.e., $+7 \mathrm{dBm}$, which fits the result derived in [15]. Comparing this IIP3 of $+10 \mathrm{dBm}$ to the IIP3 of $+11 \mathrm{dBm}$ shown in [13] (measured via wafer probing) with around $3 \mathrm{~dB}$ adjustment for the transmission-line effect around $500 \mathrm{MHz}$ RF, we may conclude that the bondwire in a packaged chip can degrade IIP3 by around $4 \mathrm{~dB}$ at $500 \mathrm{MHz}$ RF.

The measured $-3 \mathrm{~dB}$ IF BW is about $10 \mathrm{MHz}$ at $0.7 \mathrm{GHz} \mathrm{LO}$, while the designed IF BW is $10 \mathrm{MHz}$ at $0.5 \mathrm{GHz} \mathrm{LO}$. Since the IIR filter BW is determined by both the sampling frequency and the capacitor ratio according to (9), the difference is likely due to the variation of the $C_{s u}$-to- $C_{b}$ ratio caused by process spread, as they are built by different types of capacitors, i.e., $C_{s u}$ via MOM capacitor and $C_{b}$ via MOS capacitor. In an improved design, 
using the same type of capacitors for $C_{s u}$ and $C_{b}$ should be able to reduce the variation of the IF BW due to process spread.

For power consumption, the RFAs draw $2.3 \mathrm{~mA}$ and the IF test buffers take $2.4 \mathrm{~mA}$. The current consumption of the clock is $7.8 \mathrm{~mA}$ at $200 \mathrm{MHz} \mathrm{LO}$ and $10.6 \mathrm{~mA}$ at $900 \mathrm{MHz} \mathrm{LO}$. The overall power consumption for all blocks shown in Fig. 8 is less than $16 \mathrm{~mA}(19 \mathrm{~mW})$ at $1.2 \mathrm{~V}$ supply.

Overall, the presented DT-mixing downconverter shows a fair performance similar to others built via the CT-mixing approach, but is realized in a different way which is expected to benefit more from process scaling (see Section II-C). The proposed DT mixing technique maintains good quadrature demodulation and harmonic rejection over a wide channel BW.

\section{CONCLUSION}

A discrete-time mixing receiver architecture allowing wideband quadrature demodulation and wideband harmonic rejection has been presented. This makes RF sampling more suitable for software-defined radio (SDR) and cognitive radio (CR) receivers.

We showed that downconversion techniques can be classified based on: 1) the type of input signal, i.e., continuous versus discrete both in time and amplitude; and 2) the downconversion principle, i.e., mixing or sampling. Six classes of downconverters exist, five of which have been proposed before. We propose an architecture that fits in the 6th class, i.e., a DT-mixing technique that operates on continuous-amplitude but discretetime samples which can be obtained from CT-to-DT sampling. Different from mixing, sampling always changes the "observation rate" in the time domain. The suitability of CT-mixing and RF-sampling receivers for SDR has also been discussed, arguing that both can be suitable while RF-sampling can have advantages with respect to the compatibility to CMOS downscaling and $\mathrm{SoC}$ integration.

The proposed DT-mixing architecture has some similar features as CT mixing. It addresses two limitations of traditional RF-sampling receivers for SDRs operating with wide channel bandwidth: 1) quadrature demodulation and 2) harmonic rejection. Simulation shows wideband $90^{\circ}$ phase shift for quadrature demodulation without systematic channel bandwidth limitation. Oversampling and harmonic rejection relax RF pre-filtering and reduce noise and interference folding. The DT mixing concept can be realized via de-multiplexing. A proof-of-concept DT-mixing downconverter for the 0.2 -to- $0.9 \mathrm{GHz}$ RF band employing 8-times oversampling has been built in $65 \mathrm{~nm}$ CMOS. It can reject the 2 nd-to-6th harmonics by $40 \mathrm{~dB}$ typically and without systematic channel bandwidth limitation.

\section{ACKNOWLEDGMENT}

The authors would like to thank Freeband Communications for research funding, NXP Semiconductors for chip fabrication, especially Domine Leenaerts, and Gerard Wienk and Henk de Vries from University of Twente for their valuable assistance.

\section{REFERENCES}

[1] D. Jakonis et al., "A 2.4-GHz RF sampling receiver front-end in $0.18-\mu$ m CMOS," IEEE J. Solid-State Circuits, vol. 40, no. 6, pp. 1265-1277, Jun. 2005.
[2] H. Pekau and J. W. Haslett, "A 2.4 GHz CMOS sub-sampling mixer with integrated filtering," IEEE J. Solid-State Circuits, vol. 40, no. 11, pp. 2159-2166, Nov. 2005.

[3] R. B. Staszewski et al., "All-digital TX frequency synthesizer and discrete-time receiver for Bluetooth radio in 130-nm CMOS," IEEE J. Solid-State Circuits, vol. 39, no. 12, pp. 2278-2291, Dec. 2004.

[4] K. Muhammad et al., "The first fully integrated quad-band GSM/GPRS receiver in a 90-nm digital CMOS process," IEEE J. Solid-State Circuits, vol. 41, no. 8, pp. 1772-1783, Aug. 2006.

[5] R. B. Staszewski et al., "A $24 \mathrm{~mm}^{2}$ quad-band single-chip GSM radio with transmitter calibration in $90 \mathrm{~nm}$ digital CMOS," in IEEE ISSCC Dig. Tech. Papers, Feb. 3-7, 2008, pp. 208-607.

[6] F. Montaudon et al., "A scalable 2.4-to-2.7 GHz Wi-Fi/WiMAX discrete-time receiver in $65 \mathrm{~nm}$ CMOS," in IEEE ISSCC Dig. Tech. Papers, Feb. 3-7, 2008, pp. 362-619.

[7] J. Mitola, "Software radio architecture: A mathematical perspective," IEEE J. Sel. Areas Commun., vol. 17, no. 4, pp. 514-538, Apr. 1999.

[8] "The Software Defined Radio Request for Information," BellSouth, Atlanta, GA, Dec. 1995.

[9] R. Bagheri et al., "An 800-MHz-6-GHz software-defined wireless receiver in 90-nm CMOS," IEEE J. Solid-State Circuits, vol. 41, no. 12, pp. 2860-2876, Dec. 2006.

[10] V. Giannini et al., "A 2-mm² $0.1-5 \mathrm{GHz}$ software-defined radio receiver in 45-nm digital CMOS," IEEE J. Solid-State Circuits, vol. 44, no. 12, pp. 3486-3498, Dec. 2009.

[11] Z. Ru, N. A. Moseley, E. Klumperink, and B. Nauta, "Digitally-enhanced software-defined radio receiver robust to out-of-band interference," IEEE J. Solid-State Circuits, vol. 44, no. 12, pp. 3359-3375, Dec. 2009.

[12] Z. Ru, E. Klumperink, and B. Nauta, "On the suitability of discretetime receivers for software-defined radio," in Proc. IEEE ISCAS, May 27-30, 2007, pp. 2522-2525.

[13] Z. Ru, E. Klumperink, and B. Nauta, "A discrete-time mixing receiver architecture with wideband harmonic rejection," in IEEE ISSCC Dig. Tech. Papers, Feb. 3-7, 2008, pp. 322-616.

[14] "Notice for proposed rulemaking (NPRM 03 322): Facilitating opportunities for flexible, efficient, and reliable spectrum use employing spectrum agile radio technologies," Federal Communications Commission (FCC), ET Docket No. 03 108, Dec. 2003.

[15] Z. Ru, E. A. M. Klumperink, C. E. Saavedra, and B. Nauta, "A 300-800 $\mathrm{MHz}$ tunable filter and linearized LNA applied in a low-noise harmonic-rejection RF-sampling receiver," IEEE J. Solid-State Circuits, vol. 45, no. 5, pp. 967-978, May 2010.

[16] Y. Tsividis, "Digital signal processing in continuous time: A possibility for avoiding aliasing and reducing quantization error," in Proc. IEEE Int. Conf. Acoustics, Speech, and Signal Processing (ICASSP'04), May 17-21, 2004, vol. 2, pp. ii-589-592.

[17] B. Schell and Y. Tsividis, "A continuous-time ADC/DSP/DAC system with no clock and with activity-dependent power dissipation," IEEE J. Solid-State Circuits, vol. 43, no. 11, pp. 2472-2481, Nov. 2008.

[18] A. R. Shahani, D. K. Shaeffer, and T. H. Lee, "A 12-mW wide dynamic range CMOS front-end for a portable GPS receiver," IEEE J. SolidState Circuits, vol. 32, no. 12, pp. 2061-2070, Dec. 1997.

[19] B. W. Cook, A. Berny, A. Molnar, S. Lanzisera, and K. S. J. Pister, "Low-power 2.4-GHz transceiver with passive RX front-end and 400-mV supply," IEEE J. Solid-State Circuits, vol. 41, no. 12, pp. 2757-2766, Dec. 2006.

[20] M. Soer, E. Klumperink, Z. Ru, F. van Vliet, and B. Nauta, "A 0.2-to-2.0 GHz CMOS receiver without LNA achieving $>11 \mathrm{dBm}$ IIP3 and <6.5 dB NF," IEEE ISSCC Dig. Tech. Papers, Feb. 2009.

[21] E. A. M. Klumperink et al., "Cognitive radios for dynamic spectrum access-Polyphase multipath radio circuits for dynamic spectrum access," IEEE Commun. Mag., vol. 45, no. 5, pp. 104-112, May 2007.

[22] V. J. Arkesteijn, E. A. M. Klumperink, and B. Nauta, "Jitter requirements of the sampling clock in software radio receivers," IEEE Trans. Circuits Syst. II: Expr. Briefs, vol. 53, no. 2, pp. 90-94, Feb. 2006.

[23] K. Muhammad and R. B. Staszewski, "Direct RF sampling mixer with recursive filtering in charge domain," in Proc. IEEE ISCAS, May 23-26, 2004, vol. 1, p. I-577-80.

[24] A. J. Coulson, "A generalization of nonuniform bandpass sampling," IEEE Trans. Signal Process., vol. 43, pp. 694-704, Mar. 1995. 
[25] M. Valkama and M. Renfors, "A novel image rejection architecture for quadrature radio receivers," IEEE Trans. Circuits Syst. II: Expr. Briefs, vol. 51, no. 2, pp. 61-68, Feb. 2004.

[26] J. A. Weldon et al., "A 1.75-GHz highly integrated narrowband CMOS transmitter with harmonic-rejection mixers," IEEE J. Solid-State Circuits, vol. 36, no. 12, pp. 2003-2015, Dec. 2001.

[27] S. Lerstaveesin, M. Gupta, D. Kang, and B.-S. Song, "A 48-860 MHz CMOS low-IF direct-conversion DTV tuner," IEEE J. Solid-State Circuits, vol. 43, no. 9, pp. 2013-2024, Sep. 2008.

[28] F. Gatta et al., "An embedded $65 \mathrm{~nm}$ CMOS low-IF $48 \mathrm{MHz}-$ to-1 GHz dual tuner for DOCSIS 3.0," in IEEE ISSCC Dig. Tech. Papers, Feb. 2009, pp. 122-123.

[29] A. Molnar et al., "An ultra-low power $900 \mathrm{MHz}$ RF transceiver for wireless sensor networks," in Proc. IEEE CICC, Oct. 3-6, 2004, pp. 401-404.

[30] T. W. Brown, M. Hakkarainen, and T. S. Fiez, "Frequency-dependent sampling linearity," IEEE Trans. Circuits Syst. I: Reg. Papers, vol. 56, no. 4, pp. 740-753, Apr. 2009.

[31] Z. Ru, "Frequency translation techniques for interference-robust software-defined radio receivers," Ph.D. dissertation, Univ. of Twente, Enschede, The Netherlands, 2009.

[32] L. Joet et al., "Advanced 'Fs/2' discrete-time GSM receiver in 90-nm CMOS," in Proc. IEEE Asian Solid-State Circuits Conf. ASSCC 2006, Nov. 13-15, 2006, pp. 371-374.

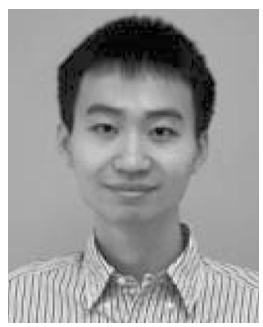

Zhiyu Ru (S'05-M'10) received the B.Eng. degree from Southeast University, Nanjing, China, in 2002, the M.Sc. degree from Lund University, Lund, Sweden, in 2004, and the Ph.D. degree from University of Twente, Enschede, The Netherlands, in 2009, all in electrical engineering.

In 2003, he was a software design engineer with Z-Com, Nanjing, China, working on WLAN products. In 2004, he did a six-month internship at Ericsson Mobile Platforms (now ST-Ericsson), Lund, Sweden, working on DVB-T receiver systems. From 2005 to 2009 , he worked as a research assistant at the IC-Design group of University of Twente on the subject of software-defined radios in CMOS. From 2009, he has been a postdoctoral researcher with the same university. He was a recipient of the ISSCC 2009 Jan van Vessem Outstanding Paper Award.

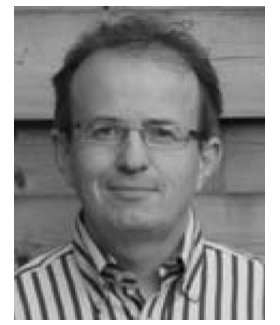

Eric A. M. Klumperink (M'98-SM'06) received the B.Sc. degree from HTS, Enschede, The Netherlands, in 1982. After a short period in industry, he joined the Faculty of Electrical Engineering of the University of Twente (UT) in Enschede in 1984, participating in analog CMOS circuit design and research. This resulted in several publications and a Ph.D. thesis, in 1997 ("Transconductance Based CMOS Circuits").

In 1998, he started as an Assistant Professor at the IC-Design Laboratory which participates in the MESA+Research Institute at the UT. His research focus changed to RF CMOS circuits for wireless and wireline communication. In 2001, he had a sabbatical at the Ruhr Universitaet in Bochum Germany, in the group of Prof. U. Langmann and Prof. H. M. Rein. Since 2006, he has been an Associate Professor at the IC Design Laboratory, participating in the CTIT Research Institute at the UT. $\mathrm{He}$ is guiding several Ph.D. and M.Sc. projects in RF CMOS research, often in cooperation with industry, while also teaching Analog and RF IC Electronics courses. He leads research projects on software-defined radio, cognitive radio and beamforming.

During 2006 and 2007, Dr. Klumperink served as an Associate Editor for the IEEE TRANSACTIONS ON CIRCUITS AND SYSTEMS II, and during 2008 and 2009 for the IEEE TRANSACTIONS ON CIRCUITS AND SYSTEMS I. He holds several patents, has coauthored more than 100 international refereed journal and conference papers, and was a corecipient of the ISSCC 2002 and the ISSCC 2009 Van Vessem Outstanding Paper Awards.

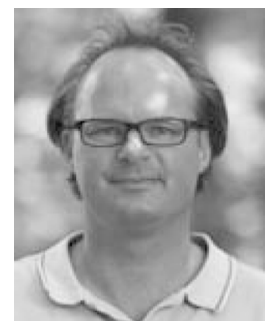

Bram Nauta (M'91-SM'03-F'07) was born in Hengelo, The Netherlands, in 1964. In 1987, he received the M.Sc. degree (cum laude) in electrical engineering from the University of Twente, Enschede, The Netherlands. In 1991 he received the $\mathrm{Ph}$.D. degree from the same university on the subject of analog CMOS filters for very high frequencies.

In 1991, he joined the Mixed-Signal Circuits and Systems Department of Philips Research, Eindhoven, The Netherlands, where he worked on high-speed AD converters and analog key modules. In 1998, he returned to the University of Twente, as full Professor heading the IC Design group, which is part of the CTIT Research Institute. His current research interest is high-speed analog CMOS circuits. He is also a part-time consultant in industry, and in 2001 he cofounded Chip Design Works. His Ph.D. thesis was published as a book: Analog CMOS Filters for Very High Frequencies (Springer, 1993), and he received the Shell Study Tour Award for his $\mathrm{Ph} . \mathrm{D}$. work.

From 1997 until 1999 he served as Associate Editor of IEEE TRANSACTIONS on Circuits AND Systems II, ANAlog and Digital Signal Processing. After this, he served as Guest Editor, Associate Editor (2001-2006), and from 2007 to 2010 as Editor-in-Chief for the IEEE JOURNAL OF SOLID-STATE CIRCUITs. He is also a member of the technical program committees of the IEEE International Solid State Circuits Conference (ISSCC), the European Solid State Circuits Conference (ESSCIRC), and the Symposium on VLSI Circuits. He was a corecipient of the ISSCC 2002 and the ISSCC 2009 Van Vessem Outstanding Paper Awards, and is an elected member of IEEE SSCS AdCom. 